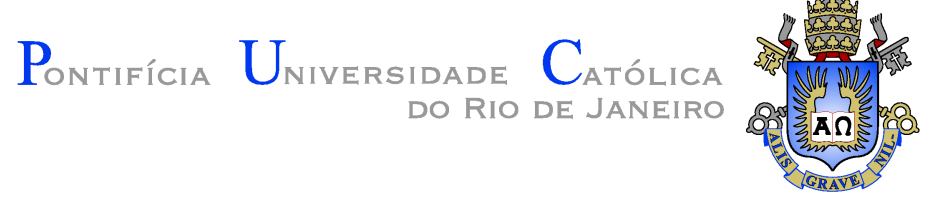

Daniel dos Santos Marques

\title{
A Decision Tree Learner for Cost-Sensitive Binary Classification
}

Dissertação de Mestrado

Dissertation presented to the Programa de Pós-Graduação em Informática of the Departamento de Informática, PUC-Rio as partial fulfillment of the requirements for the degree of Mestre em Informática.

Advisor: Prof. Eduardo Sany Laber 


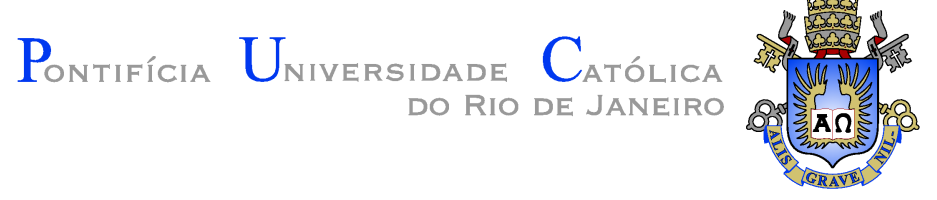

Daniel dos Santos Marques

\section{A Decision Tree Learner for Cost-Sensitive Binary Classification}

Dissertation presented to the Programa de Pós-Graduação em Informática, of the Departamento de Informática do Centro Técnico Científico da PUC-Rio, as partial fulfillment of the requirements for the degree of Mestre.

Prof. Eduardo Sany Laber

Advisor

Departamento de Informática — PUC-Rio

Prof. Ruy Luiz Milidiú

Departamento de Informática — PUC-Rio

Prof. Raúl Pierre Rentería

Departamento de Informática — PUC-Rio

Prof. Márcio da Silveira Carvalho

Coordinator of the Centro Técnico Científico - PUC-Rio

Rio de Janeiro, September 22 ${ }^{\text {nd }}, 2016$ 
All rights reserved.

\section{Daniel dos Santos Marques}

Daniel dos Santos Marques graduated from Pontifícia Universidade Católica do Rio de Janeiro in Information Systems. As an undergraduate was a member of the ICPC team.

Ficha Catalográfica

Marques, Daniel dos Santos

A decision tree learner for cost-sensitive binary classification / Daniel dos Santos Marques; advisor: Eduardo Sany Laber. - 2016.

46 f. : il. (color.); $30 \mathrm{~cm}$

Dissertação (mestrado) - Pontifícia Universidade Católica do Rio de Janeiro, Rio de Janeiro, Departamento de Informática, 2016.

Inclui bibliografia.

1. Informática - Teses. 2. Aprendizado de máquina. 3. Árvore de decisão. 4. Aprendizado sensível ao custo. I. Laber, Eduardo Sany. II. Pontifícia Universidade Católica do Rio de Janeiro. Departamento de Informática. III. Título. 


\section{Abstract}

Marques, Daniel dos Santos; Laber, Eduardo Sany (Advisor). A Decision Tree Learner for Cost-Sensitive Binary Classification. Rio de Janeiro, 2016. 46p. MSc. Dissertation Departamento de Informática, Pontifícia Universidade Católica do Rio de Janeiro.

Classification problems have been widely studied in the machine learning literature, generating applications in several areas. However, in a number of scenarios, misclassification costs can vary substantially, which motivates the study of Cost-Sensitive Learning techniques. In the present work, we discuss the use of decision trees on the more general ExampleDependent Cost-Sensitive Problem (EDCSP), where misclassification costs vary with each example. One of the main advantages of decision trees is that they are easy to interpret, which is a highly desirable property in a number of applications. We propose a new attribute selection method for constructing decision trees for the EDCSP and discuss how it can be efficiently implemented. Finally, we compare our new method with two other decision tree algorithms recently proposed in the literature, in 3 publicly available datasets.

\section{Keywords}

Machine Learning; Decision Tree; Cost-Sensitive learning. 


\section{Resumo}

Marques, Daniel dos Santos; Laber, Eduardo Sany. Uma árvore de Decisão para Classificação Binária Sensível ao Custo. Rio de Janeiro, 2016. 46p. Dissertação de Mestrado - Departamento de Informática, Pontifícia Universidade Católica do Rio de Janeiro.

Problemas de classificação foram amplamente estudados na literatura de aprendizado de máquina, gerando aplicações em diversas áreas. No entanto, em diversos cenários, custos por erro de classificação podem variar bastante, o que motiva o estudo de técnicas de classificação sensível ao custo. Nesse trabalho, discutimos o uso de árvores de decisão para o problema mais geral de Aprendizado Sensível ao Custo do Exemplo (ASCE), onde os custos dos erros de classificação variam com o exemplo. Uma das grandes vantagens das árvores de decisão é que são fáceis de interpretar, o que é uma propriedade altamente desejável em diversas aplicações. Propomos um novo método de seleção de atributos para construir árvores de decisão para o problema ASCE e discutimos como este pode ser implementado de forma eficiente. Por fim, comparamos o nosso método com dois outros algoritmos de árvore de decisão propostos recentemente na literatura, em 3 bases de dados públicas.

\section{Palavras-chave}

Aprendizado de máquina; Árvore de decisão; Aprendizado sensível ao custo. 


\section{Contents}

$\begin{array}{lll}1 & \text { Introduction } & 7\end{array}$

$\begin{array}{lll}1.1 & \text { Problem Definition } & 7\end{array}$

1.2 Our Contributions 8

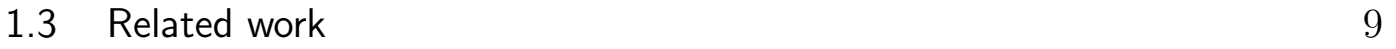

$\begin{array}{ll}1.4 & \text { Dissertation's Organization } \\ \end{array}$

2 Basic Concepts $\quad 11$

2.1 Decision Tree Construction 11

2.2 Edge Coloring for Graphs 11

$\begin{array}{lll}2.3 & \text { Bounding the Sum of Dependent Variables } & 12\end{array}$

3 The PairTree Method $\quad 13$

3.1 Evaluating the quality of attributes 14

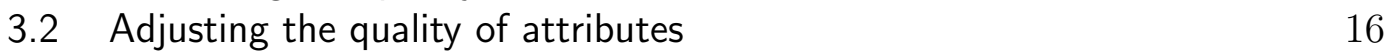

$\begin{array}{lll}3.3 & \text { Stopping Conditions } & 20\end{array}$

3.4 Implementation aspects 21

$\begin{array}{lll}3.5 & \text { Parameters Estimation } & 29\end{array}$

4 Experiments $\quad 32$

4.1 Data Sets 32

4.2 Benchmarks 34

$\begin{array}{lll}4.3 & \text { Testing Environment } & 36\end{array}$

$\begin{array}{lll}4.4 & \text { Results } & 36\end{array}$

$5 \quad$ Final remarks $\quad 42$

6 Bibliography $\quad 43$

$\begin{array}{lll}\text { A Proofs } & 45\end{array}$ 


\section{1 \\ Introduction}

Classification problems have been widely studied in the machine learning literature, generating applications in several areas such as medical diagnosis, fraud detection and others.

However in a number of scenarios misclassification costs can vary substantially. Let's take the example of a bank which needs to decide whether or not to give a loan to a customer. Clearly lending $\$ 1,000,000$ to a client which defaults his/her debt incurs a greater cost to the bank than a $\$ 1,000$ default by another client. This generates a big imbalance on misclassification costs between clients which cost insensitive classification algorithms can't handle well.

In this dissertation we study the Example-Dependent Cost-Sensitive Problem (EDCSP), where misclassification costs vary with each example, for binary classification.

\section{1 \\ Problem Definition}

Consider an universe $U$ of samples where each sample is modeled by a set of attributes $A_{1}, \ldots, A_{m}$. Each attribute $A_{i}$ can be thought as a function that maps each sample $s \in U$ into a value $A_{i}(s)$. In addition, we have two classes 0 and 1 and a cost function $C: U \times\{0,1\} \mapsto R^{+}$mapping each pair $(s, c)$ into a real number that represents the cost of a sample $s$ when it is assigned to class c.

Our goal is to learn a function $f$ that maps each sample into a class $c \in\{0,1\}$ so that $\sum_{s \in U} C(s, f(s))$ is minimized. For that, we are given a subset $S$, randomly selected from $U$, and the values $C(s, 0)$ and $C(s, 1)$ for every $s \in S$.

Here, we restrict our attention to the case where $f$ belongs to the class of decision tree functions. A decision tree $D$ for a set of samples $S$ is a rooted tree where each of its nodes is associated with an attribute and each of its leaves is associated with a class. Each edge $e$ that leaves a node, associated with an attribute $A$, is associated with a subset of the values that $A$ may assume. These subsets must be pairwise disjoint.

To classify a sample $s$ using a decision tree $D$ we follow a root to leaf path as follows: if the root of $D$ is also a leaf we assign to $s$ the class associated 
with the leaf. Otherwise, if the root of $D$ is associated with an attribute $R$, we recursively apply the procedure for the sub-tree rooted at the node we reach following the branch associated with $R(s)$.

In Figure 1.1 we show how we can classify two samples $s_{1}$ and $s_{2}$ in a decision tree using attributes $A_{1}$ and $A_{2}$.

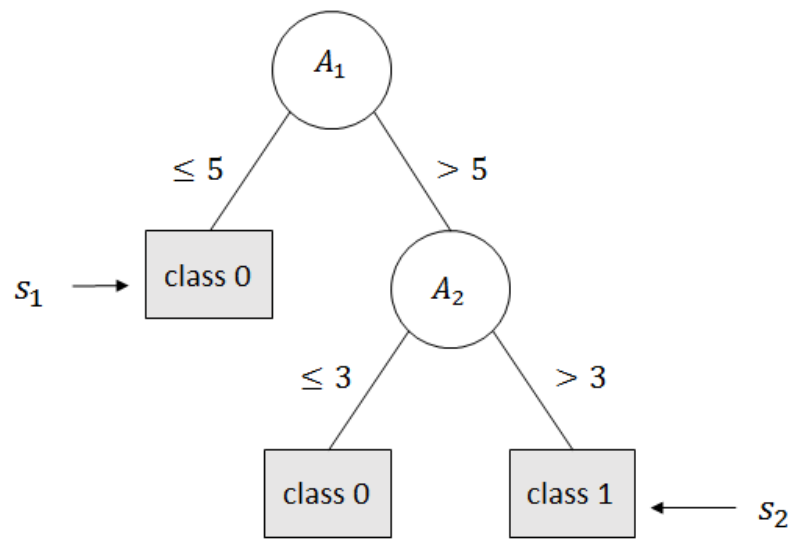

\begin{tabular}{|c|c|c|}
\hline & $A_{1}$ & $A_{2}$ \\
\hline$S_{1}$ & 2 & 10 \\
\hline$S_{2}$ & 6 & 5 \\
\hline
\end{tabular}

Figure 1.1: Decision Tree Example

One of the main motivations for using decision trees, in oppose to other methods, is that they provide a simple interpretation that is highly desirable in a number of applications.

\section{2}

\section{Our Contributions}

We propose a new decision tree method for the Example-Dependent CostSensitive Problem (EDCSP) problem, denoted by PairTree.

Our method is based on a new metric to measure the quality of an attribute split in a tree node. This new metric considers each pair of examples and measures how much is gained by splitting them into separate nodes. We also take into account how a random attribute, one which randomly splits the dataset, performs on our metric. This way we can measure how good an attribute is compared to a random attribute. This helps to avoid overfit as we can stop growing the tree if all attributes perform no significantly better than a random split.

We discuss our method implementation aspects in order to make its computation feasible for larger datasets. We use the Binary Indexed Tree (Fenwick, 1994) data structure to calculate our metric for numerical attributes.

We make an experimental study on three publicly available datasets: Direct Marketing (Moro et al., 2014), Give Me Some Credit (Kaggle.com, 2011), and PAKDD2009 (PAKDD, 2009). Our method showed superior results 
when compared to two other decision tree methods from the cost-sensitive learning literature proposed in (Aodha and Brostow, 2013) and (Bahnsen et al., 2015).

We compared our results using the Savings metric which shows how good a tree is when compared to the strategy of classifying all examples in one class.

$$
\text { Savings }=1-\frac{\text { Tree Cost }}{\text { Cost of best class }}
$$

Our PairTree method achieved the best results for both Give Me Some Credit (50.59\%) and PAKDD2009 (29.82\%) datasets, an improvement of $6 \%$ and $4 \%$ respectively when compared to the best benchmark. While for the Direct Marketing dataset our results were only $0.4 \%$ worse than the best benchmark.

\section{3}

\section{Related work}

Costs naturally occur in the classification task as it had already been noticed in Breiman's seminal book (Breiman et al., 1984). Two common sources of costs are the attributes and the misclassification. As an example, in a diagnosis procedure some blood exams may be much more expensive than others and, still in this context, classifying a patient as healthy when he is sick may be much more risky than the other direction. For a survey focused on cost-sensitive decision trees algorithm we recommend (Lomax and Vadera, 2013) in which the authors present over 50 algorithms.

Here, we focus on misclassification costs. This type of cost can be naturally introduced in 2 different ways: In the most general version, the Example-Dependent Cost-Sensitive Problem (EDCSP), the cost of classifying a sample depends on the sample and the predicted class. While in the restricted version, Class-Dependent Cost-Sensitive Problem (CDCSP), the cost depends on the true class and the predicted class. The latter is a particular case of the former as it can be seen as an EDCSP where the misclassification costs are the same for all examples. Here, however, we focus on the EDCSP.

In (Zadrozny et al., 2003), Zadrozny et al. presents techniques to address EDCSP that are based on a central theorem that shows how to change the sample distribution so that traditional classifiers 'become' cost-sensitive. These techniques differ mainly on how the samples are drawn from the new distribution. An experimental study with these methods is carried on the KDD98 and DMEF-2 datasets.

In (Aodha and Brostow, 2013), a Random Forest for the EDCSP is proposed. To build the decision trees that are used for the committee they 
propose a new impurity measure that takes into account, for each example $s$, the difference between the cost of classifying $s$ as $i$ and $j$ for each pair of classes $i$ and $j$. They compare their method with two baselines: the Gini-based Cost-Sensitive Random Forest (GCSRF) and the naive Classification Random Forest (CLRF). They report improved results for 3 multi-class computer vision datasets at a price of a modest increase in the training time.

In (Bahnsen et al., 2015), Bahnsen et. al. define a simple cost-based impurity measure for building decision trees for the example dependent cost sensitive problem. The method is compared with some state-of-the-art methods in over 3 datasets. They report that their method outperforms all the others, including decision trees, logistic regression and random forests trained with the techniques proposed by Zadrozny (Zadrozny et al., 2003) and Elkan (Elkan, 2001). It must be mentioned that the metric presented in (Bahnsen et al., 2015) had already been mentioned/proposed in other papers (Pazzani et al., 1994), (Turney, 1995), (Ling et al., 2004) and (Greiner et al., 2002) in the context of the CCDCP.

\section{4}

\section{Dissertation's Organization}

In Chapter 2 we discuss some basic concepts necessary to the understanding of our decision tree method which is presented on Chapter 3.

The experimental study is described at Chapter 4 in which we execute our method on 3 publicly available datasets, and compare it with two other decision tree benchmarks from the cost-sensitive learning literature.

Chapter 5 holds some final remarks and a conclusion to this work. 


\section{2 \\ Basic Concepts}

In this chapter we discuss some concepts that are important to understand our work.

We start by introducing a general framework of decision tree construction, which will be used later when we discuss our method. We then discuss how to bound the sum of dependent variables using the edge coloring concept from graph theory.

\section{1}

\section{Decision Tree Construction}

As described by (Murthy, 1998) there are different ways to construct a decision tree, but the most commonly used one is the greedy top-down approach.

On greedy top-down methods we start with the entire dataset and split it into two or more disjoint subsets, according to some predefined rule. We then recursively call the method on the generated subsets. We can also use a stopping rule, to decide whether or not we should continue growing the tree.

We provide a pseud-code below.

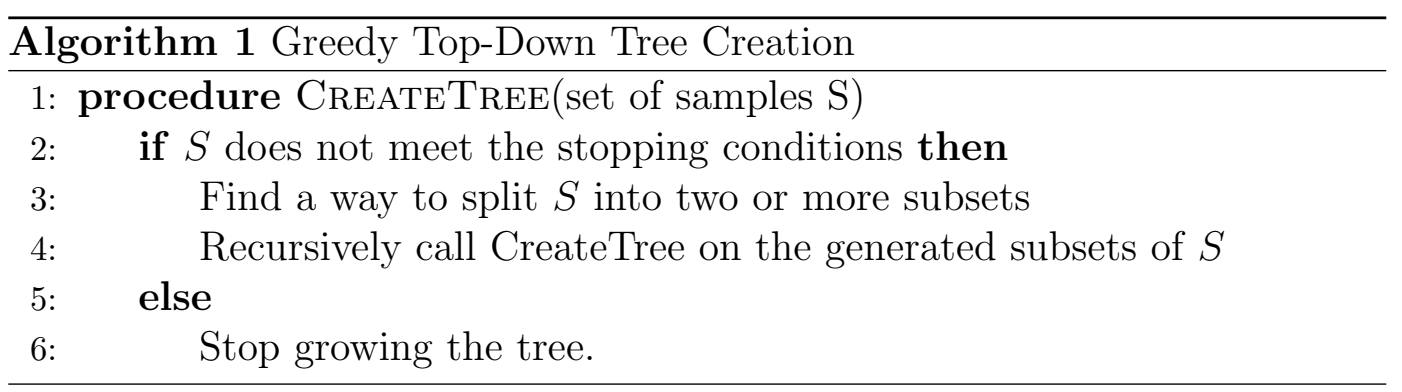

It's worth noticing that this approach can be used both for cost-sensitive and cost-insensitive classification problems.

\section{2}

\section{Edge Coloring for Graphs}

Let $G=(V, E)$ be a graph. A $k$-edge coloring for $G$ is a function that maps each edge into an integer (color) in the set $\{1, \ldots, k\}$ such that edges that share an endpoint are mapped into different colors.

The chromatic index of a graph $G$ is the minimum integer $k^{*}$ for which $G$ admits a $k^{*}$-coloring. It is known (Vizing., 1964) that the chromatic number 
of a graph is either $\Delta(G)$ or $\Delta(G)+1$, where $\Delta(G)$ is the maximum degree of the vertices in $G$. It is also known that for bipartite graphs the chromatic index is $\Delta(G)$.

\section{3}

\section{Bounding the Sum of Dependent Variables}

Let $\mathcal{Y}=\left\{Y_{1}, Y_{2}, \ldots, Y_{k}\right\}$ be a set of real valued random variables, where $Y_{i}$ takes values on the interval $\left[a_{i}, b_{i}\right]$ for $i=1, \ldots, k$. A family $\mathcal{F}$ of subsets of $\mathcal{Y}$ is a cover for $\mathcal{Y}$ if the union of all subsets of $\mathcal{F}$ is equal to $\mathcal{Y}$. A subset $\mathcal{Z} \subseteq \mathcal{Y}$ is independent if the variables in $\mathcal{Z}$ are independent. A cover $\mathcal{F}$ for $\mathcal{Y}$ is proper if all subsets of $\mathcal{F}$ are independent. Let $\chi(\mathcal{Y})$ be the size of the proper cover for $\mathcal{Y}$ with minimum cardinality.

The following theorem was proved by Janson in (Janson, 2004).

Theorem 1. (Janson, 2004) Let $X=\sum_{i=1}^{k} Y_{i}$ and let $t>0$. Then,

$$
P(X \geq E[X]+t) \leq \exp \left(\frac{-2 t^{2}}{\chi(\mathcal{Y}) \sum_{i=1}^{k}\left(b_{i}-a_{i}\right)^{2}}\right)
$$

For our study, the case where the variables $Y_{i}$ 's can be seen as edges of a complete bipartite graph $G$ and a set of variables is independent if and only if the underlying edges correspond to a matching in $G$ is of particular interest. In this case, every edge coloring for $G$ corresponds to a proper cover for $\mathcal{Y}$ and the size of the minimum proper cover for the set of variables is at most the chromatic index of $G$, which is equal to $\Delta(G)$. Hence, we can state the following corollary of Theorem 1

Corollary 1. Let $G=(V, E)$ be a bipartite graph where $E=\left\{e_{1}, \ldots, e_{m}\right\}$ and the maximum degree of $G$ is $\Delta(G)$. In addition, let $\mathcal{Y}=\left\{Y_{1}, \ldots, Y_{m}\right\}$ be set of random variables that satisfies the following property: for all $S \subseteq\{1, \ldots, m\}$, the set of variables $\left\{Y_{i}|i \in S|\right\}$ is independent if and only if $\left\{e_{i}|i \in S|\right\}$ is a matching in $G$.

Let $X=\sum_{i=1}^{m} Y_{i}$ and let $t>0$. Then,

$$
P(X \geq E[X]+t) \leq \exp \left(\frac{-2 t^{2}}{\Delta(G) \sum_{i=1}^{k}\left(b_{i}-a_{i}\right)^{2}}\right),
$$




\section{3}

\section{The PairTree Method}

In this chapter, we provide a description of our decision tree method for the two class Example-Dependent Cost-Sensitive Classification problem. The following notation is helpful for our discussion: for a set of samples $S$ and a class $\kappa$ we define

$$
C(S, \kappa)=\sum_{x \in S} C(x, \kappa)
$$

. In addition, we define

$$
S_{0}=\{x \mid x \in S \text { and } C(x, 0) \leq C(x, 1)\}
$$

and

$$
S_{1}=\{x \mid x \in S \text { and } C(x, 0)>C(x, 1)\}
$$

One of the key issues for designing decision tree learners is to define how an attribute $A$ splits the set of samples $S$ associated with a node of the tree. Our method defines partitions for nominal and numeric attributes in different ways.

Let $A$ be a nominal attribute that takes values on the domain $\left\{v_{1}, \ldots, v_{k}\right\}$ for the samples of set $S$. In our method, $A$ splits the samples of $S$ into $k$ groups $\left\{G_{1}, \ldots, G_{k}\right\}$, where $G_{i}$ contains all samples $s$ for which $A(s)=v_{i}$. On the other hand a numeric attribute with values in the range $[a, b]$, splits $S$ into two groups $\left(G_{1}^{\ell}, G_{2}^{\ell}\right)$, where $G_{1}^{\ell}$ contains all samples with values smaller than or equal to $\ell$ and $G_{2}^{\ell}$ contains all samples with values larger than $\ell$. The value $\ell$ is chosen so as to generate a partition with best possible quality according to the metric proposed in the next section.

Given a training set $S$ of $N$ samples $\left\{s_{1}, s_{2}, \ldots, s_{N}\right\}$, our method, outlined in Algorithm 2, follows a top-down approach. First, it verifies whether the set $S$ meets a stopping criterion that is also discussed further. In the positive case, we create a leaf and associate it with class 0 if $C(S, 0)<C(S, 1)$ and with class 1 , otherwise. In the negative case, the procedure selects the attribute which provides the split with best quality for the set $S$, where the quality of a split is measured by a new cost-sensitive metric explained in the next section. This attribute, say $A$, is set as the root $r$ of the decision tree. Next, for each group 
in the partition of $S$, induced by $A$, we recursively construct a decision tree and set it as a child of $r$.

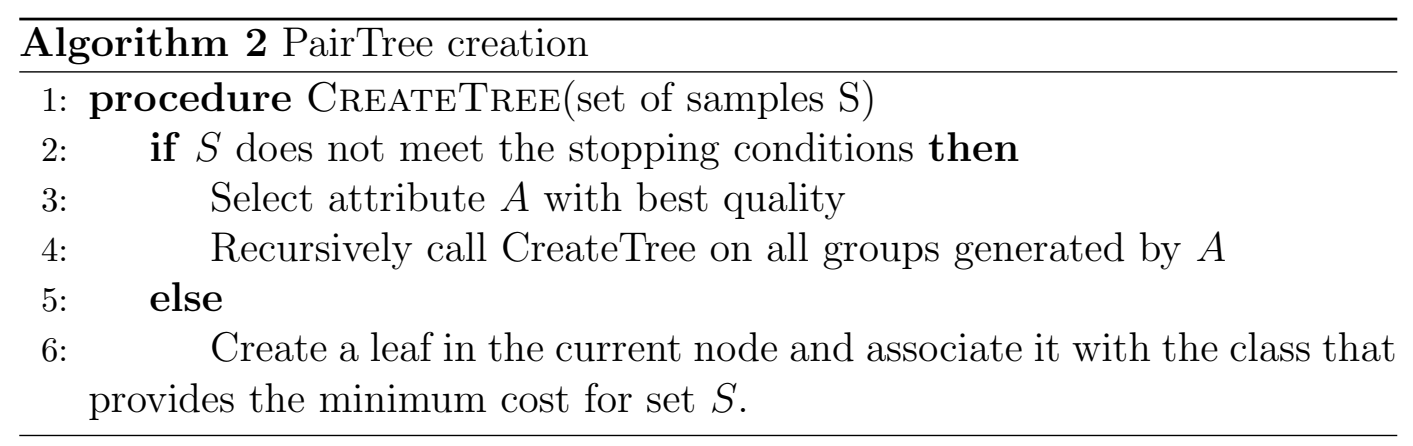

\section{1}

\section{Evaluating the quality of attributes}

In order to explain the quality of an attribute w.r.t. a set of samples $S$, we first define the gain $G(x, y)$ of separating two samples $x$ and $y$ as

$$
\begin{array}{r}
G(x, y)=\min \{C(x, 0)+C(y, 0), C(x, 1)+C(y, 1)\} \\
-(\min \{C(x, 0), C(x, 1)\}+\min \{C(y, 0), C(y, 1)\})
\end{array}
$$

This measure captures the benefit of separating $x$ and $y$ by considering the minimum possible cost when they are in the same group, which is $\min \{C(x, 0)+C(y, 0), C(x, 1)+C(y, 1)\}$ and the minimum possible cost when both are in different groups, which is $\min \{C(x, 0), C(x, 1)\}+$ $\min \{C(y, 0), C(y, 1)\})$. Note that if the class of minimum cost for $x$ and $y$ is the same then $G(x, y)=0$ because there is no gain in separating them. This generalizes the metric proposed in (Golovin et al., 2010; Cicalese et al., 2014).

The gain $\operatorname{Gain}(A, S)$ of a nominal attribute $A$ w.r.t. a set of samples $S$, is defined as the sum of $G(x, y)$ for all pairs of samples in $S$ that are separated by $A$, that is, samples that belong to different groups in the partition of $S$ induced by $A$ :

$$
\operatorname{Gain}(A, S)=\sum_{x, y \in S \mid A(x) \neq A(y)} G(x, y)
$$

For the sake of a simpler notation, whenever the context is clear we drop $S$ from $\operatorname{Gain}(A, S)$.

To motivate our metric we discuss how it compares with the impurity metric proposed in (Bahnsen et al., 2015). Let $C(S, x)$ be the cost of classifying all samples of $S$ as $x$. The gain of a binary attribute $X$ that splits $S$ into groups $A$ and $B$ is given by 


$$
\min \{C(S, 0), C(S, 1)\}-\min \{C(A, 0), C(A, 1)\}-\min \{C(B, 0), C(B, 1)\}
$$

One potential advantage of our metric with respect to this one is that it is more sensible to capture the progress made by an attribute for the classification task.

As an example, consider a set of 1000 samples formed by two groups, say $X$ and $Y$, where $X$ has 100 samples and $Y$ has 900 . The costs are either 0 or 1 where for all samples $x \in X, C(x, 0)=0$ and $C(x, 1)=1$ while for all $y \in Y$ we have $C(y, 0)=1$ and $C(y, 1)=0$.

Let us consider an attribute $A$ that splits the samples in $X \cup Y$ into two groups $X \cup Y^{\prime}$ and $Y-Y^{\prime}$, where $Y^{\prime}$ is a subset of $Y$ containing 150 samples, according to the figure below.

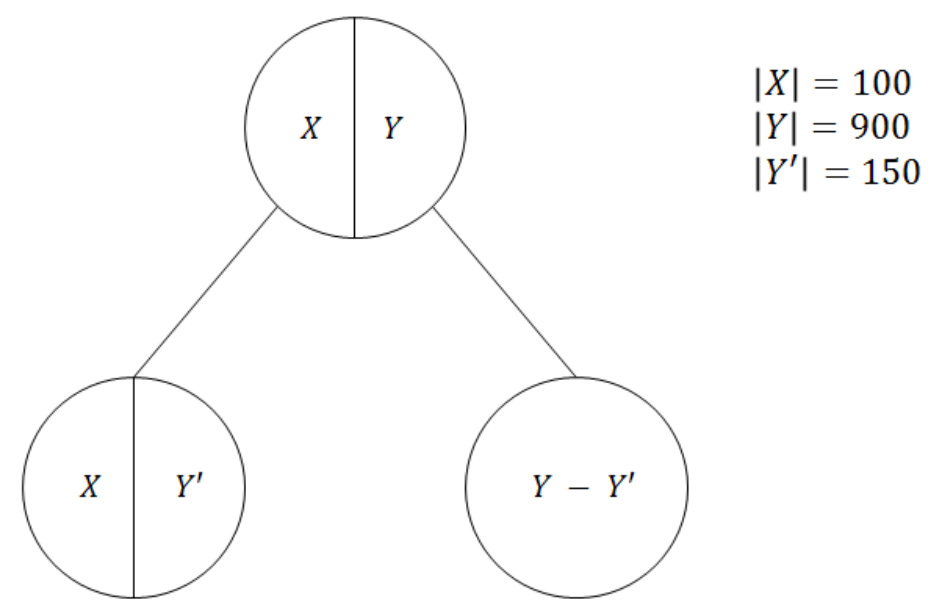

Figure 3.1: Metric Example

Attribute $A$ does a very good job in separating samples from $X$ and $Y$. However, since the minimum cost of both children and parent is achieved when using class 1 , the metric of equation (3.3) is equal to 0 indicating there is no gain in using $A$. 
$C\left(X \cup Y^{\prime}, 0\right)=750$

$C\left(X \cup Y^{\prime}, 1\right)=100$

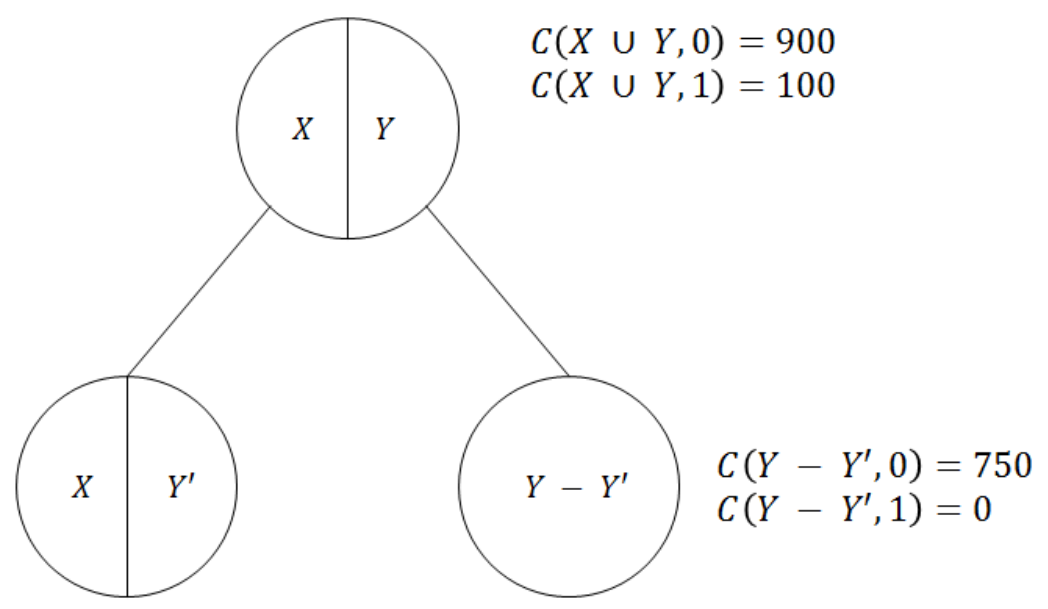

Figure 3.2: Metric Costs Example

For this example the metric proposed here can be written as the sum of $G(x, y)$ for the separated samples:

$$
\sum_{\substack{x \in X \\ y \in Y-Y^{\prime}}} G(x, y)=\sum_{\substack{x \in X \\ y \in Y-Y^{\prime}}} 1=750 \times 100=75000
$$

While the best possible attribute would've achieved a gain of $900 \cdot 100=$ 90000 .

\section{2}

\section{Adjusting the quality of attributes}

One problem with our metric is that it tends to favor attributes that may assume a large number of distinct values. This effect has been reported in the literature and there are some methods available to cope with this problem.

To illustrate this issue let's consider a database of 3650 people, with 1825 men and 1825 women, that were born in a given year. Each person is modeled by two attributes: IsTall and DayofBirth. The attribute IsTall is TRUE if the person has height larger than $1.80 \mathrm{~m}$ and FALSE, otherwise. The attribute DayofBirth is a number in the set $\{1, \ldots, 365\}$.

Our task is to classify people according to gender: male/female. Which attribute provides the best split?

The misclassification costs are either 0 or 1 , where we have $C(x, 0)=0$ and $C(x, 1)=1$ for a all men $x$ and for all women $y$ follows $C(y, 0)=1$ and $C(y, 1)=0$. Table 3.1 illustrates an example of this dataset. 


\begin{tabular}{|l|l|l|l|l|l|}
\hline Index & DayOfBirth & IsTall & $\cdots$ & $C(s, 0)$ & $C(s, 1)$ \\
\hline 1 & 64 & TRUE & $\cdots$ & 0 & 1 \\
\hline 2 & 105 & FALSE & $\cdots$ & 1 & 0 \\
\hline$\vdots$ & $\vdots$ & $\vdots$ & $\vdots$ & $\vdots$ & $\vdots$ \\
\hline 3650 & 342 & FALSE & $\cdots$ & 0 & 1 \\
\hline
\end{tabular}

Table 3.1: Example Dataset

Clearly, we expect the attribute IsTall to be more effective than DayofBirth. Let's assume there is no correlation between the day of birth and gender, and we observe exactly 5 men and 5 women with the same birth date for every day of the year. So attribute DayofBirth splits the 3650 people on the dataset according to figure 3.3

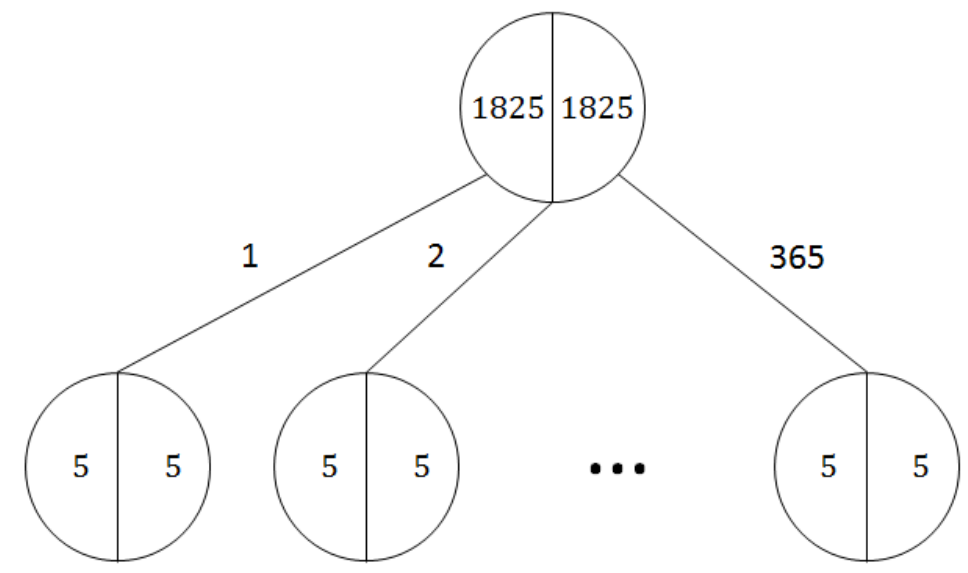

Figure 3.3: Attribute DayofBirth

To calculate our Gain metric for the DayofBirth attribute we need to count how many pairs of men and women were separated after the split. By Figure 3.3 we can see a man is separated from all women except for the 5 in his node (1820 in total). Since we have 1825 men in the datset we can write our Gain as:

$$
\text { Gain }(\text { Dayof Birth })=1820 \times 1825=3321500
$$

To calculate the Gain for attribute IsTall let's assume $30 \%(1 \%)$ of men (women) are taller than $1.80 \mathrm{~m}$. In our dataset this gives a total of 548 tall men, and 2 tall women. Figure 3.4 shows a split formed by attribute IsTall. 


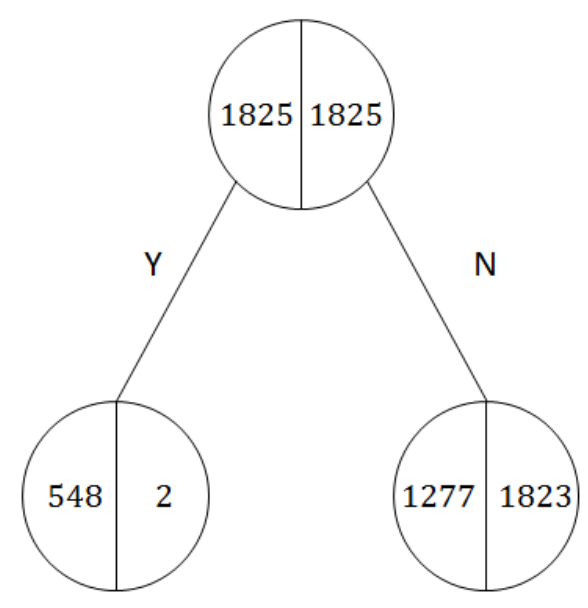

Figure 3.4: Attribute IsTall

We can calculate the Gain of IsTall using a similar approach as we used for DayofBirth, which gives us:

$$
\operatorname{Gain}(\text { IsTall })=548 \times 1823+2 \times 1277=1001558
$$

Thus, according to our metric DayofBirth is better than IsTall.

To prevent the bias towards attributes with large number of values we adjust the Gain of the partition $\mathcal{P}(S, A)$ induced by an attribute $A$ on the set of samples $S$ by comparing it with the Gain of a 'random partition' $R$ that has properties similar to $\mathcal{P}(S, A)$.

We say partition $\mathcal{P}(A, S)$ is a split, on $S$, formed by $k$ disjoint groups $G_{i}$. Where a group $G_{i}$ is the set of all samples in $S$ that assume the $k$-th value in attribute $A$.

Let $R$ be a partition of the set of samples $S$ generated according to the following process:

For $\mathrm{i}=1, \ldots,|S|$

Assign sample $i$ to group $j$ with probability $\left|G_{j}\right| /|S|$

\section{End For}

Informally, $A$ is a good split if the probability of a random partition $R$, obtained by the above process, generates a gain larger than that of $A$ is small. Let

$$
\alpha(S, A)=\operatorname{Pr}[\operatorname{Gain}(R) \geq \operatorname{Gain}(A)]
$$

where $R$ is sampled according to our process.

We would like to select the attribute $A$ such that $\alpha(S, A)$ is minimum. A problem with this approach is that is not clear how to calculate $\alpha(S, A)$ efficiently. 
As an example, one approach for estimating $\alpha(S, A)$ might consist of randomly generating several partitions, according to our process, and counting how many had a Gain greater than or equal to $\operatorname{Gain}(A)$. This is naturally a very slow solution, as it requires a great number of random draws.

Our approach to this problem makes use of Corollary 1 of Theorem 1. We need some definitions.

For a partition $R$, generated by our process, and two samples $x$ and $y$, we define the variable

$$
Y_{x, y}= \begin{cases}G(x, y), & \text { If } \mathrm{x} \text { and } \mathrm{y} \text { belongs to different groups in } R \\ 0, & \text { Otherwise }\end{cases}
$$

By using the fact that $G(x, y)=0$ if either $x, y \in S_{0}$ or $x, y \in S_{1}$ we get that

$$
\operatorname{Gain}(R)=\sum_{x, y \in S} Y_{x, y}
$$

Thus,

$$
\begin{array}{r}
\alpha(S, A)=\operatorname{Pr}[\operatorname{Gain}(R) \geq \operatorname{Gain}(A)]= \\
\operatorname{Pr}\left[\sum_{x, y} Y_{x, y} \geq \operatorname{Gain}(A)\right]
\end{array}
$$

The difficulty of bounding $\operatorname{Pr}\left[\sum_{x, y} Y_{x, y} \geq \operatorname{Gain}(A)\right]$, and as a consequence $\alpha(S, A)$, relies on the fact that the variables $Y_{x, y}$ are not independent so that Chernoff/Hoeffding's bounds cannot be used. However, Theorem 1 allows to deal with the sum of dependent variables.

To understand the dependence between our variables $Y_{x, y}$ 's it is useful to identify the variables $Y_{x, y}$ 's as edges of a complete bipartite graph $G$ with bi-partition $\left(S_{0}, S_{1}\right)$. It is not difficult to realize that a set of variables is independent if and only if the underlying edges correspond to a matching in the graph. Thus, it follows from Corollary 1 that

$\alpha(S, A)=\operatorname{Pr}[\operatorname{Gain}(R) \geq E[\operatorname{Gain}(R)]+t] \leq \exp \left(\frac{-2 t^{2}}{\max \left\{\left|S_{0}\right|,\left|S_{1}\right|\right\} \sum_{x, y \in S} G(x, y)^{2}}\right)$

where $t=\operatorname{Gain}(A)-E[\operatorname{Gain}(R)]$

Let

$$
\hat{\alpha}(S, A)=\exp \left(\frac{-2 t^{2}}{\max \left\{\left|S_{0}\right|,\left|S_{1}\right|\right\} \sum_{x, y \in S} G(x, y)^{2}}\right)
$$


be the right-hand side of the equation above.

Instead of selecting the attribute $A$ with minimum $\alpha(S, A)$, our method selects the attribute $A$ with minimum $\hat{\alpha}(S, A)$.

\section{3}

\section{Stopping Conditions}

Our method uses two parameters MaxProb and MinSamples to determine whether the decision tree construction is pruned at a given node $\nu$. In fact, the tree is pruned if one of the following conditions occurs:

i There is no attribute $A$ with $\hat{\alpha}(S, A) \leq$ MaxProb;

ii The size of a set of samples associated with $\nu$ is smaller than MinSamples

iii All samples of $S$ are in $S_{0}$ (or $\left.S_{1}\right)$

Condition (i) requires that there is an attribute that produces a rare gain in the sense that it should be much better than that of a random partition. This condition could be used alone to prune the tree because it is not reasonable to keep growing the tree if all attributes produce 'random' gains.

The reason why we also use condition (ii), however, is because we do not have a sharp estimation for $\alpha(S, A)$. To understand this issue, assume that we set MaxProb $=0.1$. Because the bound is not sharp it may happen that we are just accepting attributes whose gains are extremely rare, that is, the probability of occurring them is much smaller than 0.1 . This way we are being too restrictive.

One attempt to handle this situation is using large values for MaxProb. However, this is also risky because we could be accepting attributes that behave as random partitions, which could lead to data overfitting. Using a lower bound on the number of samples reduces the risk of overfitting the data when we are working with large values for MaxProb.

If condition (iii) is met then it's useless to make any further splits as all the samples are best classified in the same class. Therefore node $v$ should be a leaf.

At this point our method is completely specified as shows Algorithm 3. In the sequel we discuss how to implement it in an efficient way.

condition (iii) is not met 


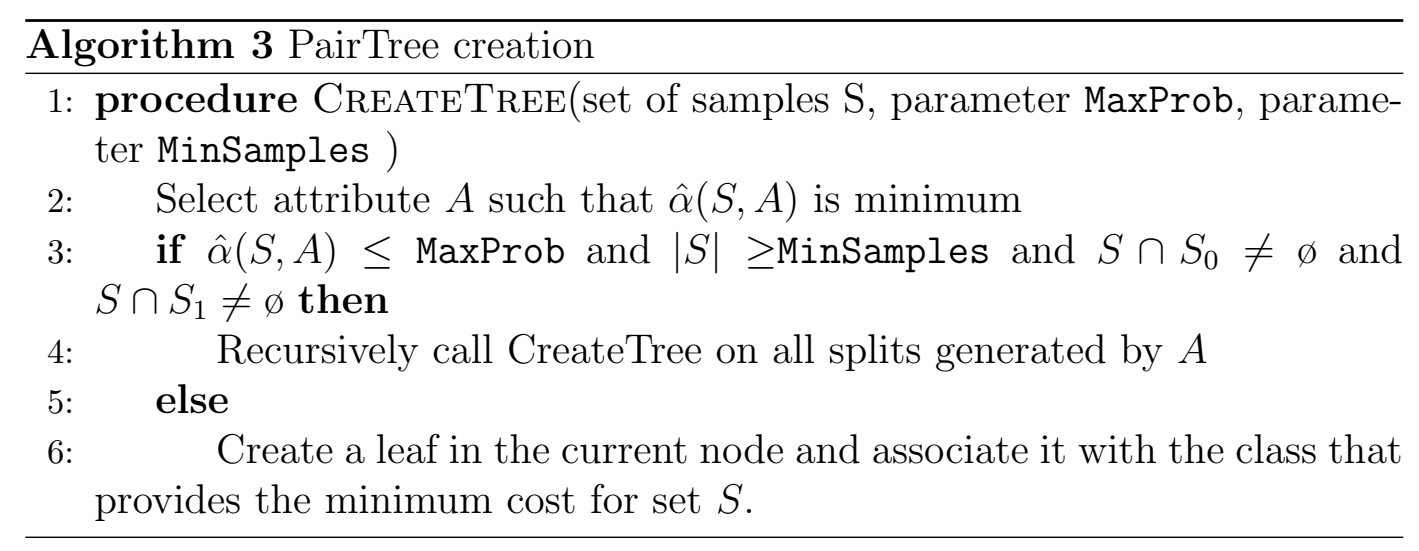

\section{4}

\section{Implementation aspects}

For an efficient implementation of our decision tree construction we need to evaluate $\hat{\alpha}(S, A)$ quickly. For that we need to calculate $\operatorname{Gain}(A)$, $\sum_{x, y \in S} G(x, y)^{2}$ and $E[\operatorname{Gain}(R)]$.

First, we consider the computation of $E[\operatorname{Gain}(R)]$. Recall that $A$ induces the partition $\mathcal{P}(A, S)=\left(G_{1}, \ldots, G_{k}\right)$ when applied to set of samples $S$. Let $p_{i}=\left|G_{i}\right| /\left(\left|G_{1}\right|+\cdots+\left|G_{k}\right|\right)$. We have that

$$
\begin{gathered}
E[\operatorname{Gain}(R)]=E\left[\sum_{x, y \in S} G(x, y)\right]=\sum_{x, y \in S} E[G(x, y)]= \\
\sum_{x, y \in S} \sum_{i=1}^{k} G(x, y) \cdot p_{i} \cdot\left(1-p_{i}\right)=\left(\sum_{x, y \in S} G(x, y)\right)\left(\sum_{i=1}^{k} p_{i} \cdot\left(1-p_{i}\right)\right)
\end{gathered}
$$

where the product $p_{i}\left(1-p_{i}\right)$ is the probability that in a random partition sample $x$ belongs to $i$-th group and sample $y$ does not belong it.

To evaluate $E[\operatorname{Gain}(R)]$ we need to compute $\sum_{x, y \in S} G(x, y)$, which can be seen as the gain of a 'perfect' attribute, say $P$, that splits the set $S$ into $S_{0}$ and $S_{1}$. Thus, if we compute $\operatorname{Gain}(P)$ efficiently we manage to compute $E[\operatorname{Gain}(R)]$ efficiently as well.

In the sequel we discuss how to efficiently compute the gain of any attribute, in particular the attribute $P$, and how to compute $\sum_{x, y \in S} G(x, y)^{2}$. The latter calculation is pretty straightforward given that we know how to compute $\operatorname{Gain}(P)$. We first consider nominal attributes and then numeric attributes. 


\subsection{1}

\section{Nominal Attributes}

We wish to calculate the Gain of a nominal $k$-valued attribute $A$ for a set of $N$ samples $S$ in an efficient manner. There is a direct algorithm which consists of trying all pairs of samples taking $O\left(N^{2}\right)$ time. We wish to speed up this process as a time complexity of $O\left(N^{2}\right)$ can be prohibitive for some large datasets.

We rewrite the Gain of an attribute $A$ in a way that will make the computation faster. First, we define the absolute difference $D(s)$ of a sample $s \in S$ as

$$
D(s)=|C(s, 0)-C(s, 1)|
$$

For simplicity whenever we refer to a sample $x(y)$ we're dealing with a sample from the set $S_{0}\left(S_{1}\right)$. The following propositions turns out to be useful.

Proposition 1. $G(x, y)=\min \{D(x), D(y)\}$

\section{Proposition 2.}

$$
\begin{aligned}
\operatorname{Gain}(A) & =\sum_{x \in S_{0}} D(x) \cdot|\{y \mid A(x) \neq A(y) \wedge D(x) \leq D(y)\}| \\
& +\sum_{y \in S_{1}} D(y) \cdot|\{x \mid A(x) \neq A(y) \wedge D(x)>D(y)\}|
\end{aligned}
$$

We develop a procedure to calculate the quantities $\mid\{y \mid A(x) \neq A(y) \wedge$ $D(x) \leq D(y)\} \mid$ for every $x \in S_{0}$ and $|\{x \mid A(x) \neq A(y) \wedge D(x)>D(y)\}|$ for every $y \in S_{1}$, described in Proposition 2, in linear time after an $O(n \log n)$ time preprocessing.

For that we create an array $D I F F$ containing all samples $s$ ordered in ascending order by $D(s)$. If two samples $x$ and $y$ have the same $D(x)$ and $D(y)$ we put $x$ first in the order.

Using vector $D I F F$ we define two new variables:

$T C_{0}^{i}=$ number of samples in $S_{0}$ in the subarray $D I F F[i: N]$

$T C_{1}^{i}=$ number of samples in $S_{1}$ in the subarray $\operatorname{DIFF}[i: N]$

We note that, for $c=0$ and $c=1$,

$T C_{c}^{i}= \begin{cases}T C_{c}^{i-1}-1 & \text { if sample } s \text { in position } i-1 \text { of vector } D I F F \text { is in } S_{c} \\ T C_{c}^{i-1} & \text { Otherwise }\end{cases}$ 
In addition, we define the set of variables

$T C V_{c, j}^{i}=$ number of samples in $S_{c}$ valued $j$ in attribute $A$ in the subarray $\operatorname{DIFF}[i: N]$

We note that

$T C V_{c, j}^{i}= \begin{cases}T C V_{c, j}^{i-1}-1 & \text { if sample } s \text { in position } i-1 \text { of vector } D I F F \text { is in } S_{c} \text { and } A(s)=j \\ T C V_{c, j}^{i-1} & \text { Otherwise }\end{cases}$

If $x \in S_{0}$ corresponds to the $i$-th element of $D I F F$ we have that

$$
|\{y \mid A(x) \neq A(y) \wedge D(x) \leq D(y)\}|=T C_{1}^{i+1}-T C V_{1, A(x)}^{i+1} .
$$

Similarly, if $y \in S_{1}$ corresponds to the $i$-th element of DIFF we have that

$$
|\{x \mid A(x) \neq A(y) \wedge D(y)>D(x)\}|=T C_{0}^{i+1}-T C V_{0, A(y)}^{i+1}
$$

By using the expressions above and Proposition 2, we can rewrite $\operatorname{Gain}(A)$ as:

$$
\operatorname{Gain}(A)=\sum_{i=0}^{N} D\left(s_{i}\right)\left(T C_{c}^{i}-T C V_{c, A\left(s_{i}\right)}^{i}\right)
$$

where $s_{i}$ is the sample in position $i$ of vector DIFF and $s_{i} \in S_{c}$.

We can now present the following algorithm to calculate $\operatorname{Gain}(A)$ :

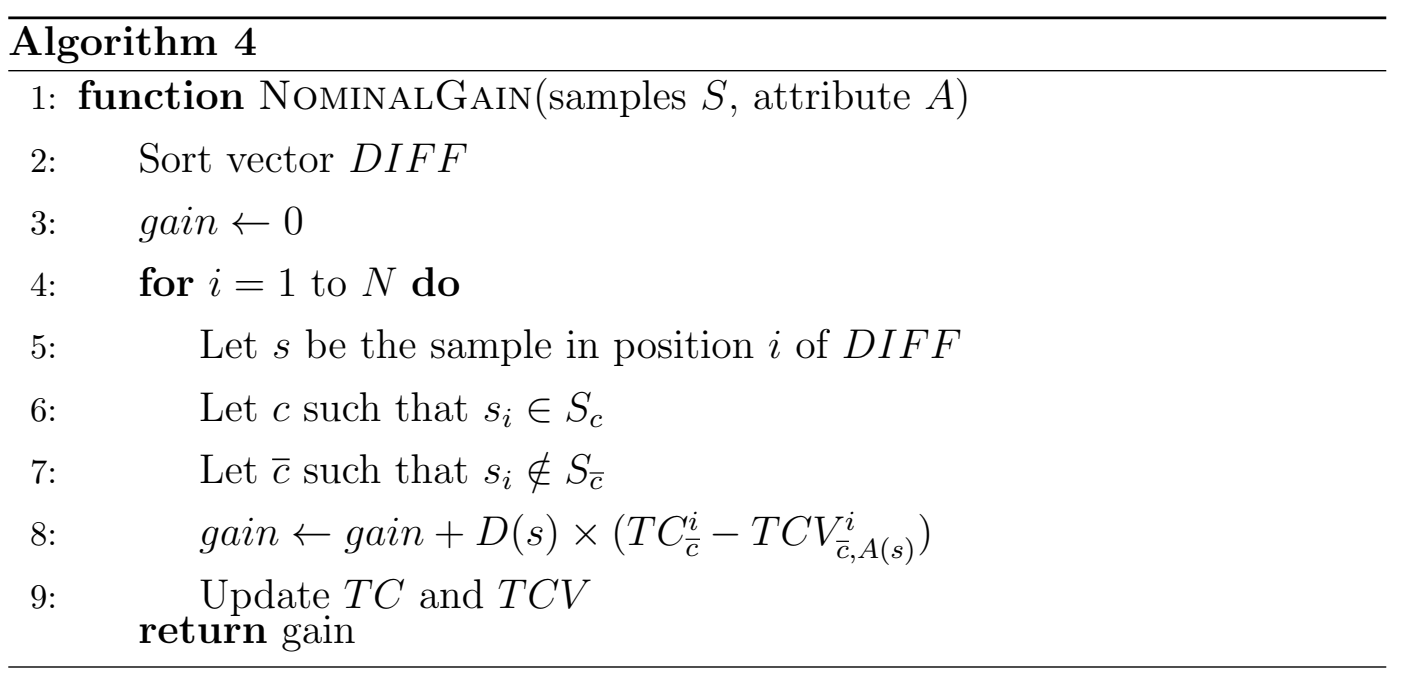

In the algorithm we can sort vector $D I F F$ in $O(N \log N)$ time so that the overall time complexity is $O(N \log N)$, which is a clear improvement over the $O\left(N^{2}\right)$ time spent by a brute force solution.

We can make one additional speed up by noting that the $O(N \log N)$ sorting of vector DIFF only needs to be made once for the whole tree construction. We can create the DIFF vector on a given non-root node 
$X$ in $O(N)$ time if we use the sorted DIFF vector for the parent of $X$. The time complexity to analyze all nominal attributes in the tree becomes $O(N \cdot \log N+\operatorname{Depth}(T) \cdot K \cdot N)$, where $K$ is the number of nominal attributes in the tree and $\operatorname{Depth}(T)$ is the depth of the tree $T$.

Finally, we note that $\sum_{x, y \in S} G(x, y)^{2}$ can be also calculated in $O(N \log N)$ time by running a slight variation of Procedure 4 in which $D(s)$ is replaced with $D(s)^{2}$ in line 8 . This variation must be executed for an attribute $P$ that splits $S$ into $S_{0}$ and $S_{1}$.

\section{4 .2}

\section{Numeric Attribute}

Numeric attributes present an additional difficulty as they can generate a large number of different partitions. Let's say we have a numeric attribute $A$ ranging in the interval $[a, b]$ for the set of $N$ samples $S$. If all samples have distinct values for attribute $A$ then we have $N$ possible choices for the splitting parameter $\ell$. Every choice of $\ell$ gives us a different binary partition $\mathcal{P}(\ell)$ formed by groups $\{\operatorname{Left}(\ell)$, Right $(\ell)\}$ with an associated Gain.

If we try all possible values for the splitting parameter $\ell$ and use the method proposed on the last subsection for each of the induced partitions, we end up with an $O\left(N^{2}\right)$ time complexity solution.

We propose an alternative solution to calculate the gain of a numeric attribute, taking $O(N \log N)$ time.

As we mentioned before, there are at most $N$ possible choices for the splitting parameter $\ell$. For simplicity whenever we say $\ell$ on this section we are referring to one of the possible values $\ell_{1}<\ell_{2}<\cdots<\ell_{N}$ that the attribute under consideration may assume.

Let's pick one possible splitting parameter $\ell_{i}(i=1 \ldots N)$ and take a deeper look on how the samples are organized. The parameter $\ell_{i}$ generates a binary split where all samples valued less or equal to $\ell_{i}$ go to the left child (group Left ${ }^{i}$ ) and the others to the right child (group Right ${ }^{i}$ ).

A sample in the left (right) child can be from either $S_{0}$ or $S_{1}$. A sample $s$ in the parent node appears on exactly one of the following groups:

- Left $t_{0}^{i}$ : Set of all samples $s$ such that $s \in S_{0}$ and $A(s) \leq \ell_{i}$.

- Left $t_{1}^{i}$ : Set of all samples $s$ such that $s \in S_{1}$ and $A(s) \leq \ell_{i}$.

- Right $t_{0}^{i}$ : Set of all samples $s$ such that $s \in S_{0}$ and $A(s)>\ell_{i}$.

- Right $t_{1}^{i}$ : Set of all samples $s$ such that $s \in S_{1}$ and $A(s)>\ell_{i}$. 
For a fast implementation we wish to efficiently compute $\operatorname{Gain}\left(A, \ell_{i+1}\right)$ for $\operatorname{Gain}\left(A, \ell_{i}\right)$. To construct $\left\{L e f t^{i+1}, \operatorname{Right}^{i+1}\right\}$ from $\left\{L e f t^{i}, \operatorname{Right}^{i}\right\}$ we need to move all samples $s$ where $A(s)=\ell_{i+1}$ from Right $^{i}$ to $L e f t^{i+1}$. Hence,

$$
\begin{array}{r}
\text { Left }^{i+1}=\text { Left }^{i} \cup M \\
\text { Right }^{i+1}=\text { Right }^{i}-M,
\end{array}
$$

where $M=\left\{s \mid s \in\right.$ Right $\left._{i} \wedge A(s)=\ell_{i+1}\right\}$.

We wish to examine how moving a sample $s \in M$ from Right $^{i}$ to Left $t^{i+1}$ changes the gain of the attribute.

The gain $\operatorname{Gain}\left(A, \ell_{i}\right)$ of numeric attribute $A$ when using the splitting parameter $\ell_{i}$ is the sum of all $G(x, y)$ such that $x$ and $y$ are separated in partition $\mathcal{P}\left(\ell_{i}\right)$. We have:

$$
\operatorname{Gain}\left(A, \ell_{i}\right)=\sum_{x \in L e f t_{0}^{i} \wedge y \in R i g h t_{1}^{i}} G(x, y)+\sum_{x \in \operatorname{Right}_{0}^{i} \wedge y \in L e f t_{1}^{i}} G(x, y)
$$

For the sake of a simpler presentation, we assume that $s$ is best classified as class 0 , that is, $s \in S_{0}$. In addition, we also assume that there is only one sample $s$ in set $M$. We discuss how to drop this assumption by the end of this section.

In order to write $\operatorname{Gain}\left(A, \ell_{i+1}\right)$ from $\operatorname{Gain}\left(A, \ell_{i}\right)$ we need to remove all contributions $G(y, s)$ from samples $y \in L e f t_{1}^{i}$ and add all contributions $G(s, y)$ from samples $y \in R i g h t_{1}^{i}$. More formally, we can write:

$$
\operatorname{Gain}\left(A, \ell_{i+1}\right)=\operatorname{Gain}\left(A, \ell_{i}\right)-\sum_{y \in \operatorname{Left}_{1}^{i}} G(y, s)+\sum_{y \in \operatorname{Right} t_{1}^{i}} G(s, y)
$$

This gives us the following pseudo-code to calculate the gain of attribute $A$ for all $\ell_{i}$. 


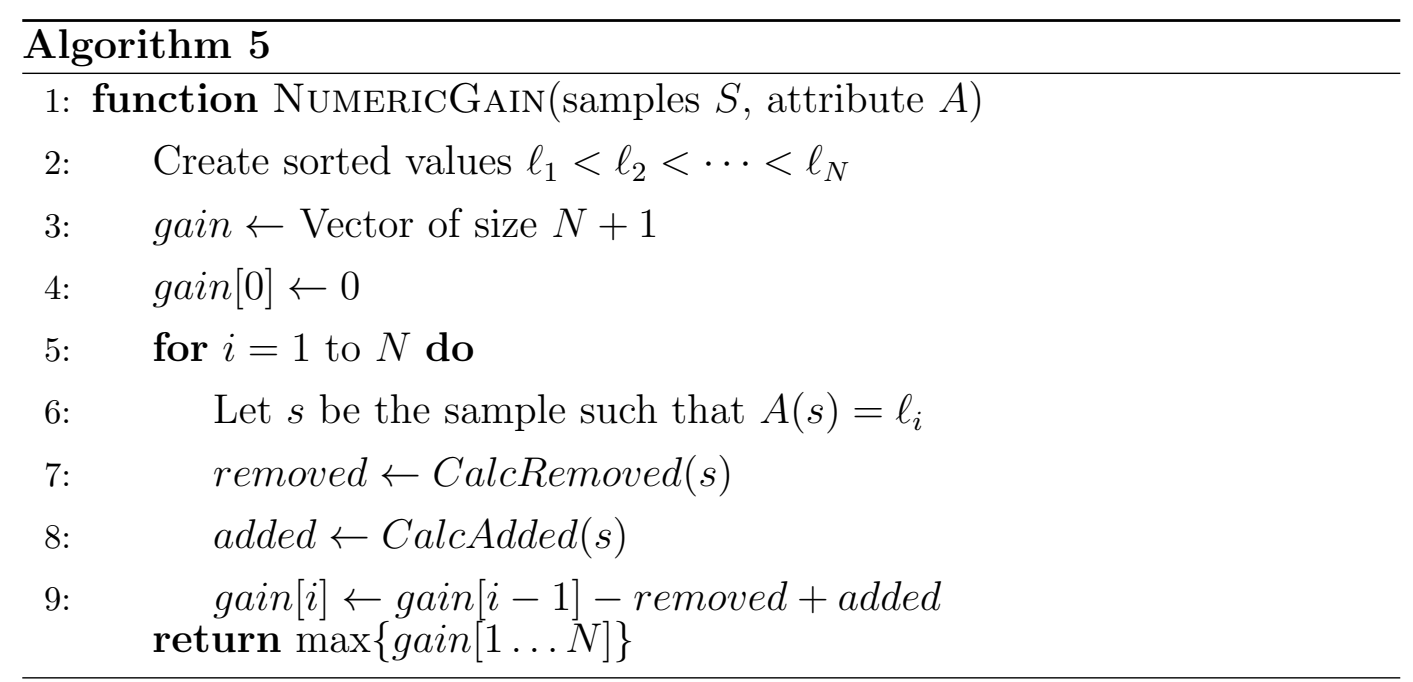

The time complexity of the above procedure heavily relies on how fast we can calculate the sums of removed and added contributions, represented by functions CalcRemoved and CalcAdded, respectively, on the pseudo-code above.

So let's examine closely the term $\sum_{y \in L e f t_{1}^{i}} G(s, y)$, representing the removed contributions on 3.13. At this point the reader should recall the definition of $D(s)$ given by equation 3.8. We can rewrite the sum as follows:

$$
\sum_{y \in L e f t_{1}^{i}} G(s, y)=\sum_{y \in L e f t_{1}^{i} \wedge D(y) \leq D(s)} G(s, y)+\sum_{y \in L e f t_{1}^{i} \wedge D(y)>D(s)} G(s, y)
$$

Let $s_{j}$ be the sample at position $j$ of $D I F F$. The following vectors will help us calculate the above sums:

$$
\begin{gathered}
D_{\text {Left }}[j]= \begin{cases}D\left(s_{j}\right) & \text { if } s_{j} \in \text { Left }_{1}^{i} \\
0 & \text { Otherwise }\end{cases} \\
\text { IndLeft }_{1}[j]= \begin{cases}1 & \text { if } s_{j} \in \text { Left } \\
0 & \text { Otherwise }\end{cases}
\end{gathered}
$$

Using the vectors above we can rewrite 3.14 as:

$$
\sum_{y \in L e f t_{1}^{i}} G(s, y)=\sum_{j=1}^{\text {pos-1 }} D \text { Left } t_{1}[j]+D(s) \cdot \sum_{j=\text { pos }+1}^{N} \text { IndLeft }_{1}[j],
$$

where pos is the index of sample $s$ in vector DIFF.

Let $\operatorname{GetSum}(\mathrm{V}, \mathrm{i}, \mathrm{j})$ be a a function that receives an array $V$ of real numbers and two positions $i$ and $j$ and returns $\sum_{k=i}^{j} V[k]$. The following pseudo-code calculates the removed contributions: 


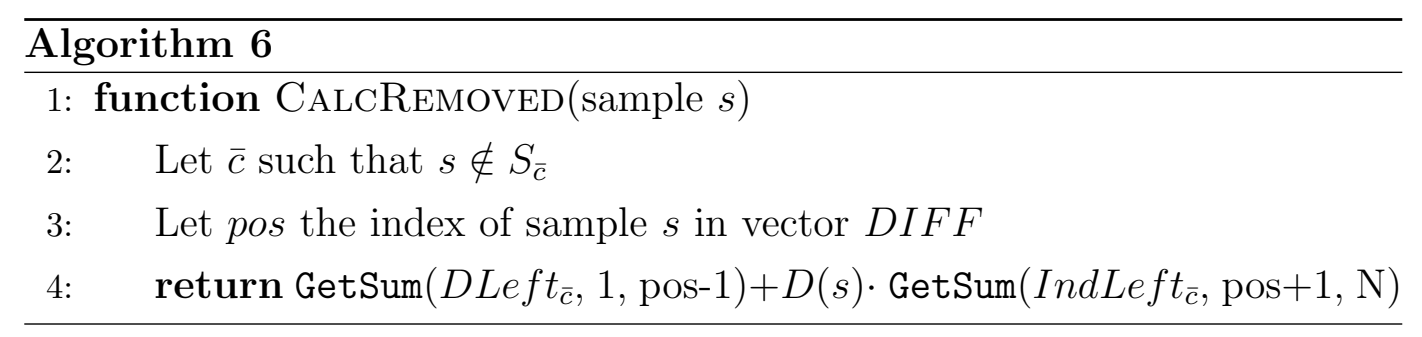

Note that the time complexity of CalcRemoved function depends on how fast we evaluate GetSum function.

Now we'll use a similar idea to write CalcAdded as a function of GetSum. We expand the added contributions term $\sum_{y \in R i g h t_{1}^{i}} G(s, y)$, rewriting it as follows:

$$
\sum_{y \in R i g h t_{1}^{i}} G(s, y)=\sum_{y \in R i g h t_{1}^{i} \wedge D(y) \leq D(s)} G(s, y)+\sum_{y \in R i g h t_{1}^{i} \wedge D(y)>D(s)} G(s, y)
$$

Again, we create 2 vectors as follows:

$$
\begin{gathered}
\text { DRight }_{1}[j]= \begin{cases}D\left(s_{j}\right) & \text { if } s_{j} \in \text { Right }_{1}^{i} \\
0 & \text { Otherwise }\end{cases} \\
\text { IndRight }_{1}[j]= \begin{cases}1 & \text { if } s_{j} \in \text { Right }_{1}^{i} \\
0 & \text { Otherwise }\end{cases}
\end{gathered}
$$

We then have

$$
\sum_{y \in R i g h t_{1}^{i}} G(s, y)=D(s) \cdot \sum_{j=p o s+1}^{N} \operatorname{IndRight}_{1}[j]+\sum_{j=1}^{\text {pos-1 }} \operatorname{DRight}_{1}[j]
$$

where pos is the index of sample $s$ in vector $D I F F$.

Finally, in pseudo-code:

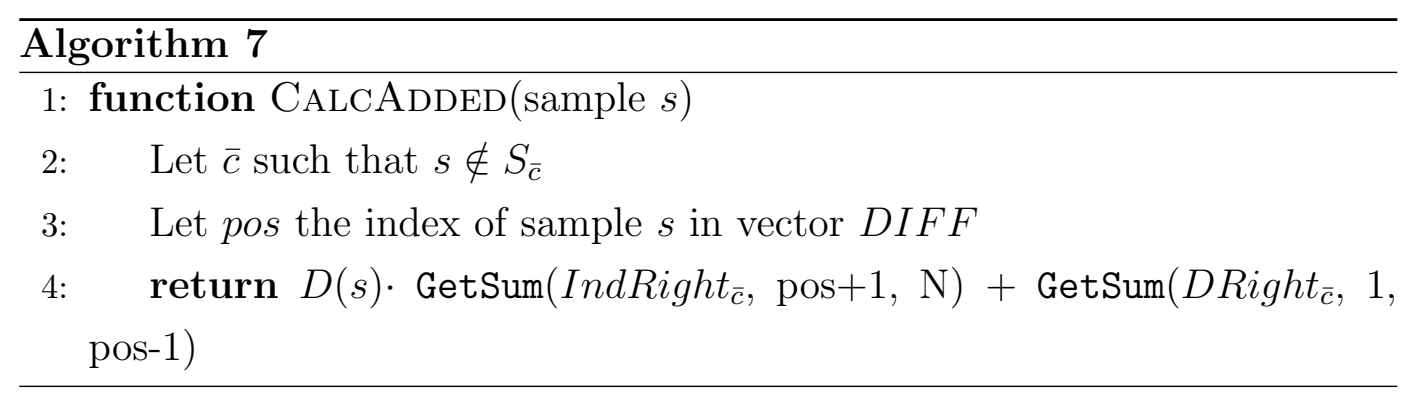

Again, the efficiency of CalcAdded relies on how fast we evaluate GetSum function.

Before proceeding the discussion, we recall that we assumed $s \in S_{0}$. The case $s \in S_{1}$ can be addressed in a similar way and it requires the usage of 
vectors $D L e f t_{0}, I_{n d L e f t_{0}}, D R i g h t_{0}$, IndRight $_{0}$ that are defined analogously to DLeft $t_{1}$ IndLeft In $_{1}$ DRight ${ }_{1}$, IndRight

Back to our discussion, right after calculating $\operatorname{Gain}\left(A, \ell_{l+1}\right)$, we have to update the vectors $D L e f t_{0}$, IndLeft $_{0}$, DRight $t_{0}$, IndRight $_{0}$ as follows: DLeft $t_{0}[$ pos $] \leftarrow D\left(s_{j}\right)$, IndLeft $t_{0}[$ pos $] \leftarrow 1$, DRight $_{0}[$ pos $] \leftarrow 0$ and IndRight $t_{0}[$ pos $] \leftarrow 0$.

The vectors DLeft $_{1}$, IndLeft $t_{1}$, DRight $_{1}$, IndRight In $_{1}$ are not updated because by assumption $s \in S_{0}$ so that they are not affected.

Algorithm 8 presents the full pseudo-code for calculating the gain of a numeric attribute.

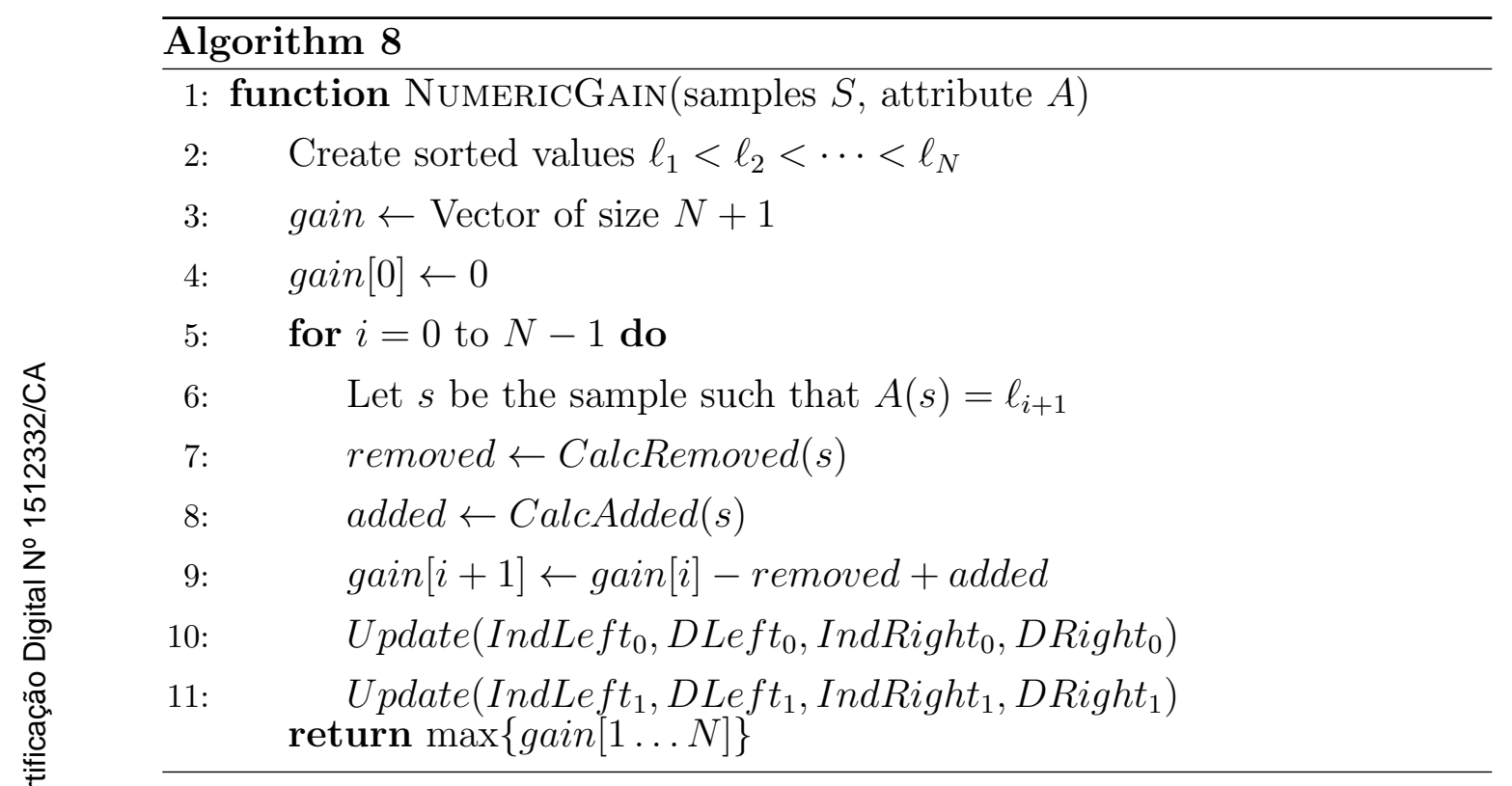

For an efficient implementation all the vectors IndLeft $t_{c}$ DLeft $t_{c}$, IndRight $_{c}$, DRight $_{c}$, for $c \in\{0,1\}$, are stored using a BIT data structure (Fenwick, 1994). Given a vector $A[1 . . n]$ of $n$ real numbers this data structure requires $O(n)$ space and supports the following operations in $O(\log n)$ time.

- UPDATE(i,x): it replaces the values of $A[i]$ with $x$;

- $\operatorname{SUM}(\mathrm{i}, \mathrm{j}):$ Return $\sum_{k=i}^{j} A[k]$

Thus, both GetSum and Update can be executed in $O(\log N)$ time while only using an extra $O(N)$ storage.

In our discussion we have assumed that there is only one sample $s$ such that $A(s)=\ell_{i+1}$ In the more general case where there are multiple samples with the same value we can execute the same algorithm with the difference that instead of returning the maximum gain among all indexes in the set $\{1,2, \ldots, n\}$, we return the maximum gain in the set $\left\{i \mid \ell_{i}<\ell_{i+1} \vee i=n\right\}$. 
This way we avoid partitions that separate samples that have the same value for attribute $A$.

In summary, we have presented an $O(N \log N)$ time algorithm to calculate the gain of a numeric attribute.

\section{5}

\section{Parameters Estimation}

Let $D_{\alpha, M}$ be the tree constructed by PairTree when it is executed with parameters MaxProb $=\alpha$ and MinSamples $=M$. In order to estimate good parameter's values we define a list of candidates $C=\left(\alpha_{1}<\alpha_{2}<\cdots<\alpha_{k}\right)$ for MinProb and a list of candidates $C^{\prime}=\left(M_{1}<M_{2}<\cdots<M_{\ell}\right)$ for MinSamples. Next, we execute a cross-validation to estimate the cost of $D_{\alpha, M}$, for each $(\alpha, M) \in C \times C^{\prime}$, and then pick the combination that yield the minimum cost (possibly after running some smoothing procedure). This approach may be very expensive for large datasets because we need to run $k \times \ell$ cross validations.

However, this procedure can be significantly accelerated by using the following simple observation:

Proposition 3. For each $(\alpha, M) \in C \times C^{\prime}$, the tree constructed by PairTree for tree $D_{\alpha, M}$ is a sub-tree of $D_{\alpha_{k}, M_{1}}$

Let's look at an example to clarify Proposition 3. Assume we want to create the trees for parameters MaxProb $=\{0.1\}$ and MinSamples $=$ $\{100,500\}$. Figure 3.5 represents the tree $D_{0.1,100}$.

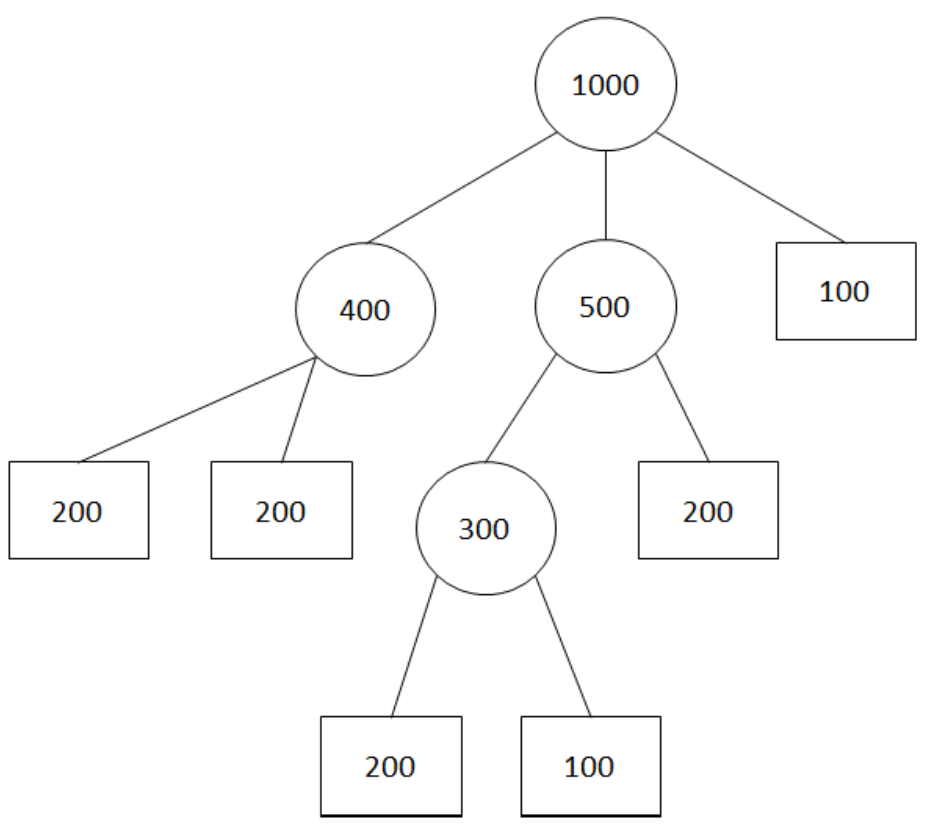

Figure 3.5: Tree for MaxProb $=0.1$ and MinSamples $=100$ 
It's simple to see we can recreate $D_{0.1,500}$ from $D_{0.1,100}$ simply by removing some nodes, resulting in Figure 3.6.

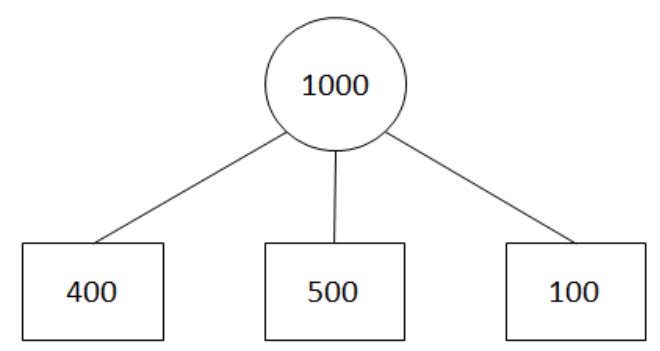

Figure 3.6: Tree for MaxProb $=0.1$ and MinSamples $=500$

The approach for speeding up the $k \times \ell$ cross-validations consists of building an augmented version of tree $D_{\alpha_{k}, M_{1}}$, denoted by $D^{\text {aug }}$. The trees $D^{a u g}$ and $D_{\alpha_{k}, M_{1}}$ have the same nodes but for each node $\nu$ in $D^{a u g}$ we store three additional information:

i $\mid$ samples $(\nu) \mid$ : the number of samples associated with $\nu$;

iii $\operatorname{class}(\nu)$, the class with minimum cost for the samples associated with $\nu$;

iii $\hat{\alpha}(\nu)=\min \{\hat{\alpha}(\operatorname{samples}(\nu), A) \mid A$ is an attribute $\}$.

This augmented tree contains all the information required to quickly classify a sample $s$ for every tree $D_{\alpha_{i}, M_{j}}$.

For storing $D^{a u g}$ we need 3 additional integers/floats per node and the additional time to build $D^{a u g}$ is $O\left(\operatorname{Depth}\left(D_{\alpha_{k}, M_{1}}\right) N\right)$, which is negligible when compared with the time complexity to build $D_{\alpha_{k}, M_{1}}$.

In order to classify a sample $s$ for the different trees $D_{\alpha, M}$ we follow a root-to-leaf path in tree $D^{a u g}$ maintaining pointers $p_{1}$ and $p_{2}$, where:

- $p_{1}$ stores the largest $i$ for which we have already visited some node $\nu^{\prime}$ with $\hat{\alpha}\left(\nu^{\prime}\right)>\alpha_{i}$ and

- $p_{2}$ stores the minimum integer $j$ for which we have already visited some node $\nu^{\prime}$ with $N\left(\nu^{\prime}\right)<M_{j}$.

Let $p_{1}^{a}$ and $p_{2}^{a}$ be, respectively, the values of $p_{1}$ and $p_{2}$ right before visiting node $\nu$. Thus, we can conclude that every tree $D_{\alpha_{i^{\prime}}}, M_{j^{\prime}}$ with $i^{\prime} \leq p_{1}^{a}$ or $j^{\prime} \geq p_{2}^{a}$ is pruned at an ancestor of $\nu$ (including $\nu$ )

Let $p_{1}^{b}$ and $p_{2}^{b}$ be, respectively, the values of $p_{1}$ and $p_{2}$ right before visiting node $\nu$.

Thus, we assign $\operatorname{class}(\nu)$ to sample $s$ for every tree $D_{\alpha_{i}, M_{j}}$ that simultaneously satisfies the formula 


$$
\left(i>p_{1}^{b}\right) \wedge\left(j<p_{2}^{b}\right) \wedge\left(i \leq p_{1}^{a} \vee j \geq p_{2}^{a}\right)
$$

We initialize $p_{1}=0$ and $p_{2}=\ell+1$ and it should be clear that $p_{1}^{a}$ and $p_{2}^{a}$ can be easily obtained given $p_{1}^{b}$ and $p_{2}^{b}$.

For classifying a sample $s$ we incur an additional $O(k \times \ell)$ time with regards to the time require to classify $s$ using tree $D_{\alpha_{k}, M_{1}}$. Since the most expensive part of the cross-validation process, in general, is the construction of the decision trees rather than the classification of the samples, we can conclude that the time spent by $k \times \ell$ cross-validations to estimate the best parameters should be slightly larger than that required for executing a single cross validation with parameters $\alpha_{k}$ and $M_{1}$. 


\section{4 \\ Experiments}

We make an experimental study of our PairTree method on 3 publicly available datasets. As way of comparison we present our results along with other decision tree benchmarks found on the literature for those datasets.

We restrict our analysis to decision tree methods as they are easily interpretable, a highly desirable quality in some applications.

\section{1}

\section{Data Sets}

The datasets were not originally cost-sensitive, however we were able to calculate the misclassification costs using methods described in the literature. These methods are based on each dataset characteristics and are described below.

\subsection{1}

\section{Direct Marketing}

This dataset contains records from clients of a Portuguese bank who were contacted by phone and offered to sign up for a financial product. The clients could either accept or deny the offer. If a client decides to adopt the product the Bank would gain an amount of cash that would depend on the client.

To calculate the cost of classifying a client in either class we use a cost matrix proposed in (Bahnsen et al., 2015) which we show here in table 4.1.

\begin{tabular}{|l|l|l|}
\cline { 2 - 3 } \multicolumn{1}{c|}{} & \multicolumn{2}{c|}{ Predicted } \\
\cline { 2 - 3 } \multicolumn{1}{c|}{} & Accept & Deny \\
\hline Accept & $C^{a}$ & Int $_{e}$ \\
\hline Deny & $C^{a}$ & 0 \\
\hline
\end{tabular}

Table 4.1: Cost matrix for sample $e$

We call the cost of contacting any client $C^{a}$ and $I n t_{e}$ the amount the bank would make if client $e$ accepts the offer.

This dataset was proposed in (Moro et al., 2014) and is publicly available on the UCI Machine Learning Repository (Lichman, 2013). The dataset originally contained 45211 instances, some of which had missing attributes. After removing instances with missing values we generated a dataset with 
37932 instances with 16 attributes which can be either nominal or numeric. In total $12.6 \%$ of clients accepted the offer.

\subsection{2}

\section{Give Me Some Credit}

This dataset contains records on clients of a bank and an indicator of whether or not they default their debt in the next 2 years. The cost for the bank of a client default (classified as bad) depends on each client.

To calculate the financial cost of a client default we used the framework proposed by (Bahnsen et al., 2015) generating a table shown 4.2.

\begin{tabular}{|l|l|l|}
\cline { 2 - 3 } \multicolumn{1}{c|}{} & \multicolumn{2}{c|}{ Predicted } \\
\cline { 2 - 3 } \multicolumn{1}{c|}{} & Good & Bad \\
\hline Good & 0 & $r_{e}+C^{a}$ \\
\hline Bad & $C l(e) \cdot L_{g d}$ & 0 \\
\hline
\end{tabular}

Table 4.2: Cost matrix for sample $e$

The cost of classifying a good client as bad is given by the amount the bank would've gained with that client $r_{e}$ plus the expected gain of lending the requested amount to another client $C^{a}$. In order to calculate the cost of classifying a bad client as good we use the assumption that clients don't default their entire debt however they only default a percentage of it, which is given by $L_{g d}$. So the cost of classifying a bad client as good is given by the amount of money requested $\mathrm{Cl}(e)$ times the average default percentage $L_{g d}$.

This dataset was provided in the Kaggle competition Give Me Some Credit (Kaggle.com, 2011) and was divided into a training set and a test set. As the competition did not provide the real classification for the test set, we can only use the training set in this work. The dataset originally contained 150000 instances, some of which had missing attributes. We removed the instances with missing values, generating a dataset with 112000 instances with 10 numerical attributes each. In total $6.74 \%$ of the clients asked for a default.

\subsection{3}

\section{PAKDD 2009}

This dataset contains records of a Brazilian credit card operator regarding their clients monthly payments. Clients that delayed any monthly payments were labeled as bad, and their misclassification costs were specific for each client. 
To calculate the cost of classifying a client in either class we selected the same cost matrix by (Bahnsen et al., 2014) used in dataset Give Me Some Credit 4.2.

This dataset was provided as part of the PAKDD 2009 competition (PAKDD, 2009). The dataset originally contained 40000 instances, some of which had missing attributes. We removed the instances with missing values, generating a dataset with 38938 instances and 26 attributes which can be either nominal or numeric. In total $19.8 \%$ of the clients are labeled as bad.

Table 4.3 presents a summary of our datasets. The column Numeric and Nominal present, respectively, the number of Numeric and Nominal attributes. The column Class Distrib. is the percentage of samples in the least frequent class.

\begin{tabular}{|c|c|c|c|c|} 
Dataset & Samples & Numeric & Nominal & Class Distrib. \\
\hline DirectMarketing & 37932 & 3 & 7 & $12.6 \%$ \\
GiveSomeCredit & 150000 & 10 & 0 & $6.74 \%$ \\
PAKDD2009 & 38938 & 11 & 9 & $19.8 \%$
\end{tabular}

Table 4.3: Summary of Datasets

\section{2}

\section{Benchmarks}

In order to compare our method and enrich our analysis we selected two decision tree algorithms from the Example-Dependent Cost-Sensitive Learning literature.

The methods in this section were originally proposed to be used only with binary splits. So to use them in datasets containing $k$-valued nominal attributes we used two approaches.

The first approach is to adapt each Benchmark to be used with nonbinary splits. We explain how this is done in each method below.

The second approach is to transform a $k$-valued nominal attribute $A n$ into $k$ new 0-1 attributes $\left\{A n_{1}, A n_{2}, \ldots, A n_{k}\right\}$ where $A n_{i}(e)=1$ if and only if $A n(e)=i$. This technique might prove too slow, and impractical, for large datasets.

\subsection{1}

\section{Aodha}

In (Aodha and Brostow, 2013), it is discussed how to use Random Forest to address the EDCSP for multiple classes. For that, they defined a costsensitive impurity measure for building the trees that compose the ensemble. 
For a sample $s$ and two classes $i$ and $j$, define $d_{i, j}^{s}=C(s, i)-C(s, j)$ if $C(s, i)-C(s, j) \geq 0$ and $d_{i, j}^{s}=0$, otherwise

In addition, define

$$
f(i, j)=\frac{\sum_{s \in S}\left(d_{i, j}^{s}\right)^{2}}{\sum_{s \in S}\left(d_{i, j}^{s}+d_{j, i}^{s}\right)}
$$

The impurity measure for two classes is defined as

$$
I_{C S}(S)=\frac{f(0,1)(1-f(0,1))+f(1,0)(1-f(1,0))}{2}
$$

The information gain for a binary attribute $A$ that splits the set $S$ into $S_{L}$ and $S_{R}$ is given by

$$
E_{\text {inf }}(S, A)=I_{C S}(S)-\left(\frac{\left|S_{L}\right|}{|S|} I_{S C}\left(S_{L}\right)+\frac{\left|S_{R}\right|}{|S|} I_{S C}\left(S_{R}\right)\right)
$$

We can make a natural modification and define the information gain for a $k$-valued attribute $A n$ which splits $S$ into $\left\{S_{1}, S_{2}, \ldots, S_{k}\right\}$.

$$
E_{\text {inf }}(S, A n)=I_{C S}(S)-\left(\frac{\left|S_{1}\right|}{|S|} I_{S C}\left(S_{1}\right)+\cdots+\frac{\left|S_{k}\right|}{|S|} I_{S C}\left(S_{k}\right)\right)
$$

In their proposal, the attribute with maximum information gain is selected. As a pruning criteria they use the height of the tree and the number of samples per leaf.

We do not use the Random Forest proposed by (Aodha and Brostow, 2013). However we use the decision tree induced by the impurity measure defined above as a benchmark.

\subsection{2}

\section{Bahnsen}

This method was proposed by (Bahnsen et al., 2014) and defines an impurity measure, similar to the one proposed by (Pazzani et al., 1994), which takes into account the cost-sensitive nature of the problem. It uses a top-down approach where in each node the attribute which maximizes a gain metric is selected to make a split, the method then recursively continues on all children.

This algorithm only uses binary splits, where for a numeric attribute $A$ in the range $[a, b]$ it tries all possible splitting parameters $l \in[a, b]$ and selects the one maximizing a gain metric, defined later.

For simplicity we use the notation $C(S, c)$ to denote the cost of classifying all samples in set $S$ as class $c$, where $c \in\{0,1\}$. 
Given a set of samples $S$ an impurity measure, which gives the minimum cost of classifying all samples in $S$ on the same class, is defined:

$$
I(S)=\min \{C(S, 0), C(S, 1)\}
$$

Using $I(S)$ the gain of a binary attribute $A$ which splits the set $S$ into $S_{L}$ and $S_{R}$ is defined, by the authors, as:

$$
\operatorname{Gain}(A)=\left(I(S)-\left(I\left(S_{L}\right)+I\left(S_{R}\right)\right)\right) / I(S)
$$

We can extend the authors Gain definition for a $k$-valued nominal attribute $A n$ which splits $S$ into $\left\{S_{1}, S_{2}, \ldots, S_{k}\right\}$.

$$
\operatorname{Gain}(A n)=\left(I(S)-\left(I\left(S_{1}\right)+\cdots+I\left(S_{k}\right)\right)\right) / I(S)
$$

The tree stops growing when no attribute generates a Gain greater than some user defined parameter MinGain.

We will not use the pruning technique proposed in (Bahnsen et al., 2014) as the authors argued the pruning gave no improvement on their results.

\section{3}

\section{Testing Environment}

All experiments were executed under the following configurations of hardware and software:

\begin{tabular}{|l|l|}
\hline Processor & Intel Core i7-4500U \\
\hline Memory & 16GB \\
\hline OS & Windows 10 \\
\hline
\end{tabular}

Table 4.4: Enviroment specs

All decision tree methods presented in this work were implemented in $\mathrm{C}++$.

\section{4}

\section{Results}

To carry on our experiments we randomly split our dataset into a training set, with $80 \%$ of the samples, and a test set containing the remaining $20 \%$.

For evaluating the quality of our methods we used the savings metric employed in (Bahnsen et al., 2015). It is defined as the ratio between the cost achieved by a tree $T$ on the set of samples $S$ and the minimum cost of classifying all samples of $S$ on the same class. In formulae, 


$$
\operatorname{Savings}(S, T)=1-\operatorname{Cost}_{T}(S) / \min \{\operatorname{Cost}(S, 0), \operatorname{Cost}(S, 1)\} \text {, }
$$

where $\operatorname{Cost}_{S}(T)$ is the total cost achieved by tree $T$ on set $S$.

\subsection{1}

\section{PairTree Parameter's estimation}

Before executing our decision tree method on the test set we need to decide the value of parameters $\alpha$ and $M$. This is done by following the procedure described in Section 3.5, using the list of values $\alpha \in\{0.05,0.1, \ldots, 1\}$ and $M \in\{30,50,100,300,500,1000,2000,3000,5000\}$, on a 10 -fold crossvalidation on the training set of each dataset.

For each combination of $\alpha$ and $M$ we calculate the average Savings of the folds validation sets.

Tables 4.5, 4.6 and 4.7 shows the Savings obtained on some $(\alpha, M)$ combinations. With the highest value of each table in bold letters.

\begin{tabular}{|c|c|c|c|c|c|c|c|c|c|c|}
\hline$\alpha / \mathrm{M}$ & 30 & 50 & 100 & 200 & 300 & 500 & 1000 & 2000 & 3000 & 5000 \\
\hline 0.1 & 46.03 & 46.03 & 46.03 & 46.03 & 46.03 & 45.88 & 45.89 & 45.10 & 44.87 & 43.04 \\
\hline 0.2 & 46.12 & 46.12 & 46.12 & 46.12 & 46.12 & 45.79 & 45.80 & 44.96 & 44.74 & 43.04 \\
\hline 0.3 & 46.30 & 46.30 & 46.30 & 46.30 & 46.29 & 45.96 & 45.95 & 44.96 & 44.74 & 43.04 \\
\hline 0.4 & 46.45 & 46.45 & 46.45 & 46.46 & 46.46 & 46.13 & 46.15 & 44.96 & 44.74 & 43.04 \\
\hline 0.5 & 46.77 & 46.80 & 46.82 & 46.83 & 46.84 & 46.36 & 46.36 & 44.96 & 44.74 & 43.04 \\
\hline 0.6 & 47.38 & 47.44 & 47.46 & 47.46 & 47.40 & 46.84 & 46.36 & 44.96 & 44.74 & 43.04 \\
\hline 0.7 & 47.44 & 47.48 & 47.50 & $\mathbf{4 7 . 5 8}$ & 47.48 & 47.17 & 46.36 & 44.96 & 44.74 & 43.04 \\
\hline 0.8 & 46.30 & 46.83 & 47.34 & 47.43 & 47.26 & 47.07 & 46.31 & 44.96 & 44.74 & 43.04 \\
\hline 0.9 & 44.51 & 45.83 & 46.74 & 47.04 & 47.24 & 47.15 & 46.42 & 44.98 & 44.76 & 43.04 \\
\hline 1 & 30.50 & 38.57 & 44.04 & 46.47 & 46.92 & 47.29 & 46.61 & 44.98 & 44.76 & 43.04 \\
\hline
\end{tabular}

Table 4.5: Cross-Validation for Direct Marketing 


\begin{tabular}{|c|c|c|c|c|c|c|c|c|c|c|}
\hline$\alpha-\mathrm{M}$ & 30 & 50 & 100 & 200 & 300 & 500 & 1000 & 2000 & 3000 & 5000 \\
\hline 0.1 & 49.97 & 49.97 & 49.97 & 49.97 & 49.97 & 49.97 & 49.97 & 49.97 & 49.97 & 48.19 \\
\hline 0.2 & 50.52 & 50.52 & 50.52 & 50.52 & 50.52 & 50.52 & 50.53 & 50.53 & 50.53 & 48.72 \\
\hline 0.3 & 50.54 & 50.54 & 50.54 & $\mathbf{5 0 . 5 4}$ & 50.54 & 50.48 & 50.51 & 50.52 & 50.58 & 48.72 \\
\hline 0.4 & 50.3 & 50.3 & 50.29 & 50.29 & 50.35 & 50.25 & 50.4 & 50.41 & 50.62 & 48.72 \\
\hline 0.5 & 50.01 & 50.03 & 49.95 & 49.99 & 49.98 & 49.9 & 50.1 & 50.23 & 50.49 & 48.65 \\
\hline 0.6 & 49.16 & 49.26 & 49.14 & 49.09 & 49.15 & 49.35 & 49.94 & 50.16 & 50.55 & 48.7 \\
\hline 0.7 & 47.45 & 47.47 & 47.37 & 47.61 & 47.87 & 48.42 & 49.51 & 50.02 & 50.43 & 48.66 \\
\hline 0.8 & 45.46 & 45.63 & 45.86 & 47.13 & 47.93 & 48.65 & 49.76 & 49.95 & 50.41 & 48.66 \\
\hline 0.9 & 41.43 & 42.81 & 43.99 & 46.78 & 47.91 & 48.89 & 49.96 & 49.96 & 50.39 & 48.66 \\
\hline 1 & 39.08 & 41.71 & 43.5 & 46.31 & 47.65 & 48.89 & 49.93 & 49.96 & 50.39 & 48.66 \\
\hline
\end{tabular}

Table 4.6: Cross-Validation for GiveMeSomeCredit

\begin{tabular}{|c|c|c|c|c|c|c|c|c|c|c|}
\hline \multirow{3}{*}{\begin{tabular}{|l|}
0.1 \\
0.2
\end{tabular}} & 30 & 50 & 100 & 200 & 300 & 500 & 1000 & 2000 & 3000 & 5000 \\
\hline & 27.31 & 27.31 & 27.31 & 27.31 & 27.31 & 27.31 & 27.31 & 27.23 & 27.23 & 24.59 \\
\hline & 27.20 & 27.20 & 27.20 & 27.20 & 27.20 & 27.20 & 27.26 & 27.23 & 27.23 & 24.59 \\
\hline 0.3 & 27.50 & 27.50 & 27.51 & 27.51 & 27.72 & 27.63 & 27.54 & 27.21 & 27.23 & 24.59 \\
\hline 0.4 & 27.42 & 27.41 & 27.44 & 27.54 & 27.75 & 27.67 & 27.59 & 27.24 & 27.23 & 24.59 \\
\hline 0.5 & 27.62 & 27.60 & 27.71 & 27.59 & 27.87 & 27.98 & 27.60 & 27.30 & 27.23 & 24.59 \\
\hline 0.6 & 26.77 & 26.79 & 27.05 & 26.94 & 27.37 & 27.99 & 27.60 & 27.30 & 27.23 & 24.59 \\
\hline 0.7 & 23.82 & 24.55 & 26.23 & 26.77 & 27.40 & 28.15 & 27.79 & 27.30 & 27.23 & 24.59 \\
\hline 0.8 & 20.31 & 21.86 & 25.16 & 25.88 & 27.61 & 28.32 & 27.91 & 27.30 & 27.23 & 24.59 \\
\hline 0.9 & 16.09 & 18.79 & 24.15 & 25.42 & 27.64 & 28.30 & 27.91 & 27.30 & 27.23 & 24.59 \\
\hline 1 & 15.79 & 18.91 & 24.11 & 25.41 & 27.64 & 28.30 & 27.91 & 27.30 & 27.23 & 24.59 \\
\hline
\end{tabular}

Table 4.7: Cross-Validation PAKDD 2009

It's interesting to observe the impact of parameters $\alpha$ and $M$ by looking at their smallest and largest values.

Large values of $\alpha$ means we are only using our Gain metric and the probability bounds proposed in Chapter 3 to select attributes in a node. But not as a way to stop growing the tree when we think the attributes are not better than the "'random attribute"', which probably causes an overfit. While for small values of $\alpha$ we might be too strict and stop growing the tree too soon, causing an underfit.

Large values of $M$ have particularly worst impact on smaller datasets such as Direct Marketing and PAKDD2009 as those combinations often create the same small tree. 
Another important aspect to look at is how the Savings on a cell behaves when compared to it's neighbors. We want to select a combination $\left(\alpha_{i}, M_{j}\right)$ with high Savings on it and around it. This shows the region shall be stable and without much random noise.

We define a simple smoothing rule to make the parameters selection.

$\operatorname{Smooth}(i, j)=\frac{S v(i, j)+0.8 \cdot(S v(i-1, j)+S v(i+1, j)+S v(i, j-1)+S v(i, j+1))}{4.2}$, where $S v(i, j)$ is the Savings of combination $\left(\alpha_{i}, M_{j}\right)$.

As a future work it makes sense to consider the effect of applying a more sophisticated procedure such as a Gaussian smoothing.

Table 4.8 shows the selected parameters using the smoothing rule for each dataset.

\begin{tabular}{|l|l|l|} 
Dataset & $\alpha$ & $\mathrm{M}$ \\
\hline Direct Marketing & 0.65 & 200 \\
Give Me Some Credit & 0.25 & 200 \\
PAKDD2009 & 0.9 & 500
\end{tabular}

Table 4.8: PairTree best parameters

Table 4.9 presents the elapsed time to execute a 10-fold cross-validation for estimating the best parameters in the training set.

\begin{tabular}{|c|c|c|c|c|} 
Dataset & Samples & Numeric & Nominal & Time (sec) \\
\hline DirectMarketing & 37932 & 3 & 7 & 20 \\
GiveSomeCredit & 112000 & 10 & 0 & 180 \\
PAKDD2009 & 38938 & 11 & 9 & 53
\end{tabular}

Table 4.9: Time required for parameter estimation

The Give Me Some Credit dataset showed to be the slowest one as it has the largest number of samples. This result is expected as we showed before on Section 3.4 the cost of calculating Gain of each numeric and nominal attributes is $O(N \log N)$, where $N$ is the number of samples.

\subsection{2}

\section{Benchmark Parameter's estimation}

To estimate the benchmarks parameters we used the same approach previously discussed for our PairTree method. The only difference being the estimated parameters. 
We believe this to be a fair approach as the authors don't mention how to make the parameter selection for their methods.

For Aodha's tree we only had one parameter in which to optimize, the minimum number of samples in each node $M$. While Bahnsen had two, MinGain which gives the minimum Gain necessary to continue growing the tree, and the minimum number of samples per node $M$.

After executing the parameter estimation procedure we used the smoothing function described in the last subsection, to get the best parameter combination. This combination was then used on the test set to get the final results.

Table 4.10 shows the selected parameters for the benchmarks using binary splits.

\begin{tabular}{|c|c|c|c|}
\cline { 2 - 4 } \multicolumn{1}{c|}{} & Aodha & \multicolumn{2}{c|}{ Bahnsen } \\
\cline { 2 - 4 } \multicolumn{1}{c|}{} & $\mathrm{M}$ & MinGain & $\mathrm{M}$ \\
\hline Direct Marketing & 1000 & 0.009 & 200 \\
\hline Give Me Some Credit & 1000 & 0.0015 & 3000 \\
\hline PAKDD2009 & 1000 & 0.005 & 50 \\
\hline
\end{tabular}

Table 4.10: Benchmarks parameters

\subsection{3}

\section{Results on the Testing Set}

Here we present our results on the test set along with the Benchmarks for comparison.

Table 4.11 shows the Savings for fitting our decision tree method on the training set, and testing it on the test set. In each dataset we show results using only binary splits labeled as "'Binary"' and using binary splits for numeric attributes and $k$-valued splits for nominal attributes labeled as " All"'. The Give Me Some Credit dataset was composed of only numeric attributes, therefore we always used binary splits.

\begin{tabular}{|c|c|c|c|c|} 
Dataset & Split & Bahnsen & Aodha & PairTree \\
\hline DirectMarketing & Binary & $47.24 \%$ & $\mathbf{4 7 . 3 6 \%}$ & $47.16 \%$ \\
DirectMarketing & All & $\mathbf{4 7 . 1 6 \%}$ & $46.82 \%$ & $46.49 \%$ \\
GiveSomeCredit & All & $47.51 \%$ & $45.41 \%$ & $\mathbf{5 0 . 5 9 \%}$ \\
PAKDD2009 & Binary & $28.37 \%$ & $28.64 \%$ & $\mathbf{2 9 . 8 2 \%}$ \\
PAKDD2009 & All & $28.37 \%$ & $28.65 \%$ & $\mathbf{2 9 . 8 2 \%}$
\end{tabular}

Table 4.11: Results on testing set 
The Direct Marketing dataset showed the least difference in Savings among the three methods and was the only dataset in which our method lost to both benchmarks.

Our method, PairTree, showed the best results for Give Me Some Credit, $6 \%$ lead over second best, and PAKDD2009 with a $4 \%$ lead.

In Table 4.12 we show the times, in seconds, necessary to create each tree before testing it on the test set.

\begin{tabular}{|c|c|c|c|c|} 
Dataset & Split & Bahnsen & Aodha & PairTree \\
\hline DirectMarketing & Binary & $<1$ & 2 & 8 \\
DirectMarketing & All & $<1$ & 1 & 2 \\
GiveSomeCredit & All & 2 & 4 & 14 \\
PAKDD2009 & Binary & $<1$ & 2 & 10 \\
PAKDD2009 & All & $<1$ & 2 & 5
\end{tabular}

Table 4.12: Tree creating times on testing set in seconds

Although our method is slower than the others, it is still fast enough to address relatively large datasets. Our metric also has the added advantage of protecting against overfit. 


\section{5 \\ Final remarks}

On this dissertation we discussed the Example-Dependent Cost-Sensitive Problem, focusing on decision tree methods. We presented recent results from the literature and how it compared to our proposed method.

Our PairTree method is based on a Gain metric which tries to capture the potential of an attribute to separate pairs of examples. We used a bound on the sum of random dependent variables, proposed on (Janson, 2004), to estimate how an attribute's Gain performs when compared to random attributes.

We achieved superior results when compared to the decision tree benchmarks (Bahnsen et al., 2015) and (Aodha and Brostow, 2013) on two datasets Give Me Some Credit and PAKDD2009. And similar results on the third dataset Direct Marketing.

As future work it's natural to think of an extension of our PairTree method to work on a multi-class classification tasks. This can probably be achieved with some changes to our Gain measure and an update on the implementation aspects in order to keep the time complexity low.

Another interesting extension to consider is the application of the PairTree method on Random Forests. This idea has the potential to increase the Savings metric. 


\section{6 \\ Bibliography}

AODHA, O. M.; BROSTOW, G. J. Revisiting example dependent cost-sensitive learning with decision trees. In Proceedings of the IEEE International Conference on Computer Vision. [S.I.: s.n.], 2013. p. 193-200. 1.2, 1.3, 4.2.1, 5

BAHNSEN, A. C.; AOUADA, D.; OTTERSTEN, B. Example-dependent costsensitive logistic regression for credit scoring. In IEEE. Machine Learning and Applications (ICMLA), 2014 13th International Conference on. [S.I.], 2014. p. $263-269.4 .1 .3,4.2 .2$

BAHNSEN, A. C.; AOUADA, D.; OTTERSTEN, B. Example-dependent costsensitive decision trees. Expert Systems with Applications, Elsevier, vol. 42, no. 19 , p. $6609-6619,2015.1 .2,1.3,3.1,4.1 .1,4.1 .2,4.4,5$

BREIMAN, L. et al. Classification and regression trees. [S.I.]: CRC press, 1984. 1.3

CICALESE, F.; LABER, E. S.; SAETTLER, A. M. Diagnosis determination: decision trees optimizing simultaneously worst and expected testing cost. In ICML. JMLR.org, 2014. (JMLR Workshop and Conference Proceedings, vol. 32), p. 414422. Available from Internet: <http://jmlr.org/proceedings/papers/v32/>. 3.1

ELKAN, C. The foundations of cost-sensitive learning. In LAWRENCE ERLBAUM ASSOCIATES LTD. International joint conference on artificial intelligence. [S.I.], 2001. vol. 17, no. 1, p. 973-978. 1.3

FENWICK, P. M. A new data structure for cumulative frequency tables. Software: Practice and Experience, Wiley Online Library, vol. 24, no. 3, p. 327-336, 1994. $1.2,3.4 .2$

GOLOVIN, D.; 0001, A. K.; RAY, D. Near-optimal bayesian active learning with noisy observations. In LAFFERTY, J. D. et al. (Ed.). NIPS. Curran Associates, Inc, 2010. p. 766-774. Available from Internet: <http://papers.nips.cc/book/advances-in-neural-information-processingsystems-23-2010>. 3.1

GREINER, R.; GROVE, A. J.; ROTH, D. Learning cost-sensitive active classifiers. Artificial Intelligence, Elsevier, vol. 139, no. 2, p. 137-174, 2002. 1.3 
JANSON. Large deviations for sums of partly dependent random variables. RSA:

Random Structures Algorithms, vol. 24, 2004. 2.3, 1, 5

KAGGLE.COM. Give Me Some Credit - Kaggle. 2011. Available from Internet: <https://www.kaggle.com/c/GiveMeSomeCredit/>. 1.2, 4.1.2

LICHMAN, M. UCI Machine Learning Repository. 2013. Available from Internet: <http://archive.ics.uci.edu/ml >. 4.1.1

LING, C. X. et al. Decision trees with minimal costs. In ACM. Proceedings of the twenty-first international conference on Machine learning. [S.I.], 2004. p. 69.1 .3

LOMAX, S.; VADERA, S. A survey of cost-sensitive decision tree induction algorithms. ACM Computing Surveys (CSUR), ACM, vol. 45, no. 2, p. 16, 2013. 1.3

MORO, S.; CORTEZ, P.; RITA, P. A data-driven approach to predict the success of bank telemarketing. Decision Support Systems, Elsevier, vol. 62, p. 22-31, 2014. $1.2,4.1 .1$

MURTHY, S. K. Automatic construction of decision trees from data: A multidisciplinary survey. Data mining and knowledge discovery, Kluwer academic publishers, vol. 2, no. 4, p. 345-389, 1998. 2.1

PAKDD. 13th Pacific-Asia Knowledge Discovery and Data Mining conference. 2009. Available from Internet: <http://sede.neurotech.com.br/PAKDD2009>. 1.2, 4.1.3

PAZZANI, M. et al. Reducing misclassification costs. In Proceedings of the Eleventh International Conference on Machine Learning. [S.I.: s.n.], 1994. p. $217-225.1 .3,4.2 .2$

TURNEY, P. D. Cost-sensitive classification: Empirical evaluation of a hybrid genetic decision tree induction algorithm. Journal of artificial intelligence research, vol. 2, p. 369-409, 1995. 1.3

VIZING., V. G. On an estimate of the chromatic class of a p-graph (in russian). Diskret. Analiz, vol. 3, p. 23-30, 1964. 2.2

ZADROZNY, B.; LANGFORD, J.; ABE, N. Cost-sensitive learning by costproportionate example weighting. In IEEE. Data Mining, 2003. ICDM 2003. Third IEEE International Conference on. [S.I.], 2003. p. 435-442. 1.3 
A

\section{Proofs}

Proposition 1. $G(x, y)=\min \{D(x), D(y)\}$

Proof.Assuming $x \in S_{0}$ and $y \in S_{1}$ we can rewrite $G(x, y)$ as

$$
\begin{array}{r}
G(x, y)=\min \{C(x, 0)+C(y, 0), C(x, 1)+C(y, 1)\} \\
-C(x, 0)-C(y, 1)
\end{array}
$$

Let's consider two cases:

i)

$$
\begin{gathered}
D(x) \leq D(y) \\
C(x, 1)-C(x, 0) \leq C(y, 0)-C(y, 1) \\
C(x, 1)+C(y, 1) \leq C(y, 0)+C(x, 0)
\end{gathered}
$$

Applying A.4 to A.1

$$
G(x, y)=C(x, 1)+C(y, 1)-C(x, 0)-C(y, 1)
$$

$$
G(x, y)=C(x, 1)-C(x, 0)
$$

As $x \in S_{0}$ therefore $C(x, 1)>C(x, 0)$, follows

$$
G(x, y)=D(x)
$$

ii)

$$
\begin{gathered}
D(x)>D(y) \\
C(x, 1)-C(x, 0)>C(y, 0)-C(y, 1) \\
C(x, 1)+C(y, 1)>C(y, 0)+C(x, 0)
\end{gathered}
$$

Applying A.9 to A.1

$$
\begin{gathered}
G(x, y)=C(x, 0)+C(y, 0)-C(x, 0)-C(y, 1) \\
G(x, y)=C(y, 0)-C(y, 1)
\end{gathered}
$$

As $y \in S_{1}$ therefore $C(y, 0)>C(y, 1)$, follows

$$
G(x, y)=D(y)
$$


Now we can write $G(x, y)$ as

$$
G(x, y)=\min \{D(x), D(y)\}
$$

\section{Proposition 2.}

$$
\begin{aligned}
\operatorname{Gain}(A) & =\sum_{x \in S_{0}} D(x) \cdot|\{y \mid A(x) \neq A(y) \wedge D(x) \leq D(y)\}| \\
& +\sum_{y \in S_{1}} D(y) \cdot|\{x \mid A(x) \neq A(y) \wedge D(x)>D(y)\}|
\end{aligned}
$$

Proof.Assuming $x \in S_{0}$ and $y \in S_{1}$.

$$
\begin{gathered}
\operatorname{Gain}(A)=\sum_{A(x) \neq A(y)} G(x, y) \\
=1 / 2 *\left(\sum_{x} \sum_{y \mid A(x) \neq A(y)} G(x, y)+\sum_{y} \sum_{x \mid A(x) \neq A(y)} G(x, y)\right)
\end{gathered}
$$

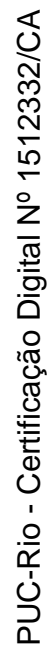

We'll consider pairs $(x, y)$ where $D(x) \leq D(y)$ in the first sum and the other pairs on the second sum

$$
=\sum_{x} \sum_{y \mid A(x) \neq A(y) \wedge D(x) \leq D(y)} G(x, y)+\sum_{y} \sum_{x \mid A(x) \neq A(y) \wedge D(x)>D(y)} G(x, y)
$$

Applying Proposition 1

$$
\begin{gathered}
=\sum_{x} \sum_{y \mid A(x) \neq A(y) \wedge D(x) \leq D(y)} D(x)+\sum_{y} \sum_{x \mid A(x) \neq A(y) \wedge D(x)>D(y)} D(y) \\
=\sum_{x} D(x) \cdot|\{y \mid A(x) \neq A(y) \wedge D(x) \leq D(y)\}| \\
+\sum_{y} D(y) \operatorname{cdot}|\{x \mid A(x) \neq A(y) \wedge D(x)>D(y)\}|
\end{gathered}
$$

